\title{
Interface Characteristics of Polystyrene Melts in Free-standing Thin Films and on Graphite Surface from Molecular Dynamics Simulations
}

\author{
Sanghun Lee, ${ }^{+,+}$Alexey V. Lyulin, ${ }^{+}$Curtis W. Frank, ${ }^{++}$and \\ Do Y. Yoon ${ }^{*,+,++}$
}

Keywords: polystyrene films; free-standing films; polymer-graphite interface; segment density profile; phenyl distribution; phenyl orientation; chain orientation; thin film Tg 


\begin{abstract}
:
Interface characteristics of polystyrene (PS) melts in free-standing thin films and on a graphite surface were investigated by molecular dynamics simulations employing an explicit all-atom force field. The calculated surface tension is in good agreement with experiment, which provides good support for the force field parameters employed. In the polymer/vacuum free-surface region, the density profile exhibits an enrichment of phenyl groups relative to the backbone alkyl groups at the outermost low-density free surface, but this free surface is followed by a layer of relatively depleted phenyls and enriched alkyls of ca. $7 \AA$ thickness. In the free surface, the phenyl-ring normal vectors and backbone chain vectors are both preferentially oriented along the film surface, in agreement with available experiments. At the polymer/graphite interface, the backbone chain vectors are strongly oriented along the graphite surface whereas the orientation distribution of phenyl-ring normal vectors exhibits two maxima along the nearly parallel $\left(20^{\circ}\right)$ and the perpendicular direction to the graphite-surface normal. A densely packed structure is formed at the PS-graphite interface, which strongly decreases the segmental chain mobility, in contrast to the enhanced segmental mobility in the free-surface region.
\end{abstract}




\section{Introduction}

Recently, polymer thin films have attracted a great deal of interest due to their increasing importance in diverse technological applications such as adhesives, lubricants, protective coatings, reverse-osmosis membranes, etc. In this regard, the key properties of polymer thin films are critically dependent on the interfacial characteristics at the polymer/solid and polymer/air interfaces over the length scales of less than ca. $1 \mathrm{~nm}$ [1]. Therefore, many experimental and simulation studies have been carried out in the last two decades on the polymer/solid interfaces as well as the polymer/air free surfaces. These studies were also motivated in large part by the important observation that the glass transition temperature $\left(T_{g}\right)$ of polymer thin films could be significantly lower than that of the bulk state $[2,3]$. As a possible origin of such a depression of $\mathrm{T}_{\mathrm{g}}$ in thin films, Kajiyama et al. first reported the existence of a more mobile region with lower $\mathrm{T}_{\mathrm{g}}$ at the polymer/air interface as a result of their measurements of the surface viscoelastic characteristics of polystyrene films of various molecular weights [4,5]. Their early results were confirmed by recent spectroscopic experiments that provided the detailed characteristics of the more mobile region in the free surface of polymer films [6].

Moreover, the polymer/solid substrate interfacial interactions are also known to play an important role in determining the $\mathrm{T}_{\mathrm{g}}$ of polymer thin films, as reported early [7-9]. Therefore, both the polymer/air free surface and polymer/solid interface strongly influence the important physical properties of polymer thin films. While the polymer/air free surface is well defined, the polymer/solid interface varies a great deal depending upon the nature of polymer/solid substrate interactions, as demonstrated by early experiments on polystyrene films on normal silicon oxide versus hydrogenated silicon oxide surfaces [7]. In this regard, a polymer/graphite interface is of current interest since polymer-carbon (graphite) fiber composites are widely employed in many important technological applications, including critical aerospace structures. More recently, 
graphene-polymer nanocomposite films are being actively investigated as a means of taking advantage of unique electronic properties of graphene for key technological applications such as transparent electrodes for electronic devices [10].

The structural characteristics of polymer thin films have been investigated by employing various experimental techniques including X-ray scattering [11], near-edge X-ray absorption fine structure (NEXAFS) spectroscopy [12], X-ray photoelectron spectroscopy (XPS) [13], secondary ion mass spectroscopy (SIMS) [13], and scanning probe microscopy (SPM) [14]. In addition, sum frequency generation (SFG) spectroscopy has been applied as a very powerful experimental technique to measure both the buried polymer/solid interface and the free polymer/air interface $[15,16]$. Since Guatam et al. first reported SFG investigations of the polystyrene (PS)/sapphire interface [17], further SFG studies have been carried out on various polymer/solid interfaces, including polystyrene on glass [18] and quartz [19], poly(methyl methacrylate) on sapphire [20], poly(n-alkyl acrylate)s on sapphire [21], and several biopolymers on solid substrates [22,23]. The results of these studies unveiled the buried polymer structures in the vicinity of solid substrates and clearly showed that the polymer/solid interface structure was strongly dependent on the characteristics of the polymer-solid surface interactions.

Chain dynamics in confined polymer melts is another important topic of the investigations on polymer thin films. Soles et al. investigated the dynamics of substrate-supported polymer thin films using incoherent neutron scattering and found that the mean square atomic displacement in polymer melts was reduced as the film thickness decreased due to the tight molecular packing caused by the thin-film confinement [24-26]. Moreover, Inoue et al. reported that, from inelastic neutron scattering experiments on a $20 \mathrm{~nm}$-thick glassy polystyrene film, the chain mobility in the direction perpendicular to the film surface was slightly greater than that in the parallel direction [27]. 
Computer simulation studies are very helpful in understanding the polymer thin films since they can provide the molecular origins of the structure and properties observed by experiments. Furthermore, using computer simulations, one can predict interesting characteristics of polymer thin films that could be measured subsequently by more advanced experiments. For example, the study of anisotropic chain mobility in polymer thin films, which was shown experimentally by Inoue et al. [27], had been motivated by an earlier simulation study of polymer thin films [28]. Therefore, many computer simulations on the polymer/vacuum and the polymer/solid interfaces have been performed over the last thirty years. In the early days, mostly coarse-grained models, such as the simple lattice model [29-31], freely-jointed hard sphere model [32-34], and Lennard-Jonnes (LJ) bead-spring chain model [35-39], were employed, followed by application of united-atom models [40-48]. The earlier studies showed the general features of polymer thin films and interfaces, but they had critical limitations in that they were unable to include accurate information about the detailed chemical structures of various polymers and their interactions at various surfaces. Such shortcomings have been improved by recent advances in computational capability and simulation algorithms, allowing large scale simulations on the polymer/vacuum [49] and the polymer/solid [50-52] interfaces with detailed consideration of explicit atomistic structures and interactions.

In the present work, we performed molecular dynamics (MD) simulations of polystyrene (PS) melts in free-standing thin films and on a graphite substrate using an explicit all-atom model [53]. Our choice of PS films was motivated, firstly, by the experimental observations that among all the polymers investigated so far, PS films exhibit the most pronounced depression of $T_{\mathrm{g}}$ in thin films and the thickest mobile layer in the free-surface region in the temperature range below the $T_{g}$ of the bulk sample [6,54]. Therefore, explicit all-atom molecular simulations of PS melts promise to yield potentially valuable insights into the molecular origin of the decreased $T_{g}$ in thin 
PS films. In this regard, our explicit-atom MD simulations of PS surfaces are also intended to check the general validity of united-atom MD simulations of PS thin films [48], which have recently been employed to investigate the thickness dependence of $T_{g}$ of free-standing PS films [55]. Secondly, PS chains are comprised of a very high fraction (75\%) of aromatic carbons and thus provide a unique opportunity to investigate the detailed characteristics and consequences of important aromatic-aromatic interactions in polymer-graphite composites and polymer-graphene nanocomposites [10].

Here, we first present the surface tension of PS melts calculated from the virial equation of the pressure tensor and thereby ascertain the validity of explicit-atom force fields for PS chains. Then we discuss the density profiles of overall atoms versus phenyl atoms and the orientation characteristics of backbone chain vectors versus phenyl-ring normal vectors in both the freesurface region and in the interface with the graphite surface, in comparison with available experiments. Finally, the segmental chain dynamics in the free-surface region and in the graphite surface region are compared with those in the bulk-like region.

\section{System specifications and simulation/computation methods}

MD simulations were performed on melts of atactic polystyrene (PS) at $450 \mathrm{~K}$, which is well above its experimental $\mathrm{T}_{\mathrm{g}}$ of $373 \mathrm{~K}$, following the simulation methods described in detail in the previous studies of polymethylene films [40] and polytetrafluorene (PTFE) films [49]. The simulation box has 20 molecules and each molecule has 15 repeating units, with the stereochemical configuration of each repeat unit chosen randomly as meso or racemic $[48,55]$. Prior to the thin film simulation, the equilibrium bulk phase was simulated in order to obtain PVT properties. In doing so, we first performed NPT MD simulations (fixed number of particles, pressure, and temperature) for a cubic simulation box with periodic boundary conditions in all 
directions [Figure 1(a)]. After the bulk phase was fully equilibrated by monitoring the density and chain conformations $[40,49,55]$, the simulation box was elongated along the $z$-direction by about 3 to 4 times without scaling the coordinates of atoms, which subsequently generates free surfaces at both sides $\left(L_{x}: 37.34 \AA, L_{y}: 37.34 \AA, L_{z}: 120.0 \AA\right)$. Using these coordinates as input, NVT MD simulations (fixed number of atoms, volume and temperature) were performed applying periodic boundary conditions in all directions [Figure 1(b)]. The MD simulations for such free-standing thin films were conducted for 24 ns, which suffices to obtain accurate ensemble averages of the properties of our interest; this is ascertained by the estimated Rouse time of ca. $7 \mathrm{~ns}$ from the previous work [56] as well as the monitored equilibration of the density profiles and the surface tension. Upon obtaining the properties of our interest in free-standing thin films, we introduced a semi-infinite graphite substrate on one free surface. In order to match the surface of the basal plane of graphite, we slightly adjusted the $x$ - and $y$-edge lengths and made the simulation box orthorhombic $\left(L_{x}: 39.36 \AA, L_{y}: 38.34 \AA, L_{z}: 120.0 \AA\right)$. The periodic boundary conditions were then applied only in the $x$-and $y$-directions [Figure 1(c)].

We used the explicit-atom force field of Smith et al. [53] for the intermolecular and intramolecular interactions of PS chains. From the bulk simulations, we obtained the density at $450 \mathrm{~K}$ of $0.984 \mathrm{~g} / \mathrm{cm}^{3}$. It is very close to the estimated value $\left(0.981 \mathrm{~g} / \mathrm{cm}^{3}\right)$ from the equation-ofstate obtained by the experiments [57]. In addition, using the same force field parameters Ayyagari et al. reproduced the equilibrium structure factors in good agreement with the X-ray scattering experiments [56]. Moreover, He et al. showed that segmental chain dynamics of PS melts obtained from the MD simulations were in agreement with the NMR experiments [58]. These prior results demonstrate that the force field employed in the present work satisfactorily describes the molecular interactions in real PS melts. 
For the interactions between the graphite substrate and the PS atoms we used Steele's potential function [59]. Using the symmetry in the crystalline substrate, he showed that the graphite substrate may be represented by a set of Lennard-Jones (LJ) carbon atoms and proposed the potential energy function between an atom and the graphite substrate given by

$V(r)=V_{o}(z)+\sum_{n>0} V_{n}(z) f_{n}(s 1, s 2)$

where $V_{o}(z)$ and $V_{n}(z)$ are given by

$V_{o}(z)=\frac{4 \pi \sigma^{6} \varepsilon}{A} \sum_{p=0}^{\infty}\left(\frac{2}{5} \frac{\sigma^{6}}{(z+p \Delta d)^{10}}-\frac{1}{(z+p \Delta d)^{4}}\right)$

$V_{n}(z)=\frac{2 \pi \sigma^{6} \varepsilon}{A}\left[\frac{\sigma^{6}}{30}\left(\frac{g_{n}}{2 z}\right)^{5} K_{5}\left(g_{n} z\right)-2\left(\frac{g_{n}}{2 z}\right)^{2} K_{2}\left(g_{n} z\right)\right]$

In equations (2) and (3), $\sigma$ and $\varepsilon$ are the size and the well-depth parameters, respectively, of the LJ interaction between the polymer atoms and the graphite carbons. $\Delta d$ is the spacing between the basal planes of graphite (=3.4 $\AA), K_{2}(x)$ and $K_{5}(x)$ are the modified Bessel functions of the second kind and the $g_{n}$ s are the numerical coefficients. The parameters $s 1$ and $s 2$ define the $x$ and the $y$-coordinates of the projection of a polymer atom on the first basal plane of graphite relative to the surface unit cell (they range from 0 to 1). Detailed functional forms of $f_{n}(s 1, s 2) \mathrm{s}$ and $g_{n}$ s are listed in reference Error! Reference source not found.. In our simulations, the 10-4 sum in equation (2) is performed explicitly over the first 63 underlying layers and the remaining contribution is accounted for by smearing the graphite carbons in all three directions resulting in a 9-3 potential form:

$V_{o}(z)=\frac{4 \pi \sigma^{6} \varepsilon}{A} \sum_{p=0}^{63}\left(\frac{2}{5} \frac{\sigma^{6}}{(z+p \Delta d)^{10}}-\frac{1}{(z+p \Delta d)^{4}}\right)+\frac{4 \pi \sigma^{6} \varepsilon}{3 A \Delta d}\left(\frac{2}{15} \frac{\sigma^{6}}{(z+64 \Delta d)^{9}}-\frac{1}{(z+64 \Delta d)^{3}}\right)$ 
We obtained LJ $\sigma$ and $\varepsilon$ parameters using Lorentz-Berthelot combining rules from Jorgensen et al.'s force fields [60] for the PS atoms and Steele's force fields [59] for the graphite atoms.

We used the Martyna-Tuckermann-Klein thermostat $[61,62]$ to maintain the temperature at the desired value and the reference system propagator algorithm as the integrator [63]. In order to account for the charge interactions, we employed the conventional Ewald sum technique for the bulk and the free-standing PS thin film simulations. For the PS thin film on graphite, which is the system with periodicity in 2-dimension, we used a technique developed by Csajka and Seidel [64]. Their method was originally proposed by Lekner [65] and modified by Sperb [66]. In the multiple time-step scheme [67], the time step was set to 2 fs for long-range interactions (electrostatic and van der Waals interactions) and 0.5 fs for short-range interactions (stretching, bending and torsional interactions). The cut-off radius for the van der Waals interaction was $8 \AA$, and thus the long-range corrections to the pressure or corresponding virials were considered. The Verlet neighbor list was used to save computer time [68].

The surface tension is calculated from the corresponding microscopic expression using the atomic virials as given in the following expression $[69,70]$

$$
\gamma=\frac{1}{2 L}\left\langle 2 P_{z z}-P_{x x}-P_{y y}\right\rangle=\frac{1}{2 A}\left\langle 2 W_{z z}-W_{x x}-W_{y y}\right\rangle
$$

In equation (5), $P_{\alpha \alpha}$ is the diagonal component of the pressure tensor, and $L$ is the size of the square surface. The surface tension is rewritten in terms of the virials in the last expression where $A$ is the surface area, and $W_{\alpha \alpha}$ is the $\alpha$-component of the atomic viral given by

$$
W_{\alpha \alpha}=\sum_{i}^{N} \sum_{a \in i}^{m}\left(\overrightarrow{f_{i}^{a}}\right)_{\alpha}\left(\overrightarrow{r_{i}^{a}}\right)_{\alpha}
$$


where $N$ is the number of molecules, $m$ is the number of atoms in a molecule, $\left(\overrightarrow{f_{i}^{a}}\right)_{\alpha}$ is the $\alpha$ component of the total force exerted on the atom $a$ of the molecule $i$ and $\left(\overrightarrow{r_{i}^{a}}\right)_{\alpha}$ is the $\alpha$ component of its position vector. However, since we used the Ewald sum method, there is a reciprocal space term that cannot be expressed by equation (6). To calculate this term, we used Alejandre et al.'s expression [71].

The contribution of the long-range correction term for the van der Waals interactions to the surface tension is not negligible [72]. This term can be evaluated using the Kirkwood-Buff equation [69] for the region beyond the cut-off and assuming the pair correlation function in this region to be unity. In addition, due to the PS/vacuum and PS/graphite interfaces, the local density around an atom is asymmetric along the $z$-axis. Hence, a self-consistent correction term to the force in the $z$-direction was accounted for by applying the density profile. The detailed equations for these long-range corrections for the surface tension and the force were given in the reference 40.

\section{Results and discussion}

\section{Free-standing PS thin film}

Surface tension. From the free-standing thin film simulations, we obtained the PS surface tension at $450 \mathrm{~K}$ of $34.5( \pm 2.1) \mathrm{dyn} / \mathrm{cm}$. Although this estimated value is greater by $\sim 18 \%$ than the experimental result of $29.2 \mathrm{dyn} / \mathrm{cm}$ [73], such a difference is not significant compared with the other surface tension estimations for polymers $[40,43,45,49]$. The discrepancy between simulation and experiment may be attributed to two possible sources. The first is the potential inaccuracy of the force field. As mentioned above, Smith et al's force field [53] employed in the 
present study describes the bulk states very well; however, the surface tension is very sensitive to the interactions among the small number of molecules in the surfaces. Thus, for a precise calculation, the force field should describe very accurately the vapor phase as well as the liquid phase. To determine whether the force field is sufficiently accurate to treat the vapor phase, however, we would need to perform simulations for phase equilibrium. Even if the force field were valid for the phase equilibrium calculation, the present simulation method could still produce an incorrect surface tension since we only included pair-wise additive terms for the van der Waals interactions in equation (6). In this regard, Barker showed from the Monte Carlo simulations of noble gases that using only the pair-wise potential terms overestimated their surface tensions [74]. Thus, for a better prediction, the three-body interaction perturbations need to be included. However, obtaining better estimations of surface tensions through development of more accurate force fields and/or including three-body perturbations would require separate detailed investigations, which are beyond the scope of the present study. Under the circumstances, we consider the force field employed to be quite satisfactory.

Segment density profiles. Figure 2 shows the segment density profiles in a free-standing PS thin film of $\sim 37 \AA$ thickness at $450 \mathrm{~K}$. The overall density profile for all atoms shows that the profile is flat in the middle of the film and that it decays smoothly in the free-surface region. Compared to the overall density profile, that of phenyl atoms is highly enhanced in the free surface and it is depleted significantly in the interfacial region adjacent to the surface. Since the total density of this interfacial region is nearly identical to that of the bulk, the density profile of the backbone alkyl atoms exhibits a concomitant enrichment in this interfacial region of ca. $7 \AA$ thickness. The major significance of this phenyl-depleted interfacial region at the free surface of PS films is discussed separately in a recent united-atom simulation study on the thicknessdependence of $T_{g}$ of PS thin films, which also considers the relative importance of chain-end 
segregation in decreasing the $\mathrm{T}_{\mathrm{g}}[55]$. As compared with the corresponding results from the united-atom simulations on free-standing PS thin film, the extent of phenyl depletion seems to be slightly more pronounced in the explicit-atom simulations; likewise, the chain-end segregation effects (noticeable in Figure 1(c)) are similar [55].

Segment orientation profiles. The orientational order parameter is used to investigate the orientational characteristics of chain segments defined as

$$
P_{2}(z)=\frac{1}{2}\left(3\left\langle\cos ^{2} \theta\right\rangle-1\right)
$$

where $\theta$ is the angle between the direction of the designated vector and the surface normal vector (vector 1 in Figure 3(a)). The value of the order parameter is 1 when the designated vectors are perfectly oriented perpendicular to the surface, $-\frac{1}{2}$ when they are perfectly aligned parallel to the surface, and 0 when they are randomly oriented. In Figure 3(b), two kinds of orientational order parameters are shown. One is that of the backbone chain vectors (vector 2 in Figure 3(a)) and the other is that of the phenyl-ring normal vectors (vector 3 in Figure 3(a)). The order parameter of the backbone chain vectors is negative in the surface region and is close to zero in the middle region. This means that the backbone chain vectors tend to orient parallel to the film surface while they are randomly oriented in the middle region. The order parameter of the phenyl-ring normal vectors resembles that of the backbone chain vector, indicating that the phenyl-ring planes at the free surface are preferentially oriented perpendicular to the film surface. Combining the density profiles and the orientational order parameters, the phenyl groups are enriched at the free surface with the phenyl-ring planes pointing outward. This structural property of the PS melt surface is in good qualitative agreement with SFG experiments [17,18] and NEXAFS experiments [75], although the experiments were performed on glassy PS films. Again, 
the segment orientation profiles are in good agreement with the united-atom simulations of PS [55].

\section{PS thin film on graphite}

Segment/atom density profiles. Figure 4(a) shows the segment density profiles of a thin film of PS melt on graphite at $450 \mathrm{~K}$. At the free-surface region, the density profiles of all the segments are well reproduced, in agreement with the free-standing thin film simulations. However, at the graphite interface, they show a very high total density layer of up to $\sim 7 \AA$ due to the favorable interaction energy between aromatic phenyls of PS chains and graphite atoms. For a detailed examination of the PS/graphite interface region, the scaled-up density plots of respective carbons in a monomer unit are shown in Figure 4(b). A significant point to observe is that at the PS/graphite interface two kinds of backbone carbon atoms (C3 and C4 in Figure 4(b)) are clearly distinguishable, i.e., the $\mathrm{C} 3$ atoms are in contact with the graphite surface while the $\mathrm{C} 4$ atoms are located slightly away from the surface. At the free surface, the $\mathrm{C} 4$ atoms are located slightly outward relative to $\mathrm{C} 3$, as pictured in Figure 3(a). Another interesting point is that the density profiles of the carbons of phenyl rings show two split peaks in the graphite interface region $(\mathrm{C} 1$ : $\sim 3.8, \sim 6.0 \AA$ and $\mathrm{C} 2: \sim 3.5, \sim 5.0 \AA$ ). This indicates that the phenyl rings may have two distinctly populated orientations, as shown in detail by the following segment orientation profiles.

Segment orientation profiles. Figure 5(a) shows the orientational order parameter in the PS thin film on graphite at $450 \mathrm{~K}$. At the free surface both the orientational order of the backbone chain vectors and that of phenyl-ring normal vectors are well reproduced to match those of the free-standing thin films. However, at the graphite interface both types of segment vectors are very highly ordered. The backbone chain vectors at the graphite interface are strongly oriented along the graphite surface while the phenyl-ring normal vectors show a strong orientation normal 
to the surface at $\sim 3.5 \AA$, followed by a strong orientation parallel to the surface at $\sim 5.7 \AA$. Because the location $(z)$, in equation (7), of the phenyl-ring normal vectors in the order parameter plot is defined as the center of phenyl ring, the vectors oriented normal to the surface are sampled at a shorter distance from the graphite surface than the vectors oriented parallel to the surface. Due to the overlap of different peaks in Figure 5(a) it is difficult to determine which of the two phenyl-ring plane orientations is preferred - parallel (left in Figure 5(b)) or perpendicular (right in Figure 5(b)) to the interface.

Figure 6 shows the angular distribution of the phenyl-ring normal vectors with respect to the surface normal. Herein we divided the film into three regions, as drawn in the top part of Figure 6: Region I for the PS/graphite interface, Region II for the middle of the film and Region III for the PS/vacuum interface. The graphite interface (I) is defined as the region from the top plane of the graphite to the location where the all-atom density shows a minimum on the graphite side of the film, and the vacuum interface (Region III) is defined as the region where the density is less than $90 \%$ of the bulk density on the vacuum side of the film. As shown in Figure 6 , in the middle of the film (Region II) the orientation of phenyl-ring planes is rather uniform while at the free surface (Region III) the phenyl-ring normal vectors show a maximum along the direction parallel to the surface $\left(\theta=90^{\circ}\right)$. In contrast, at the graphite interface (Region I) there are two maxima with the phenyl-ring planes oriented nearly parallel $\left(\theta=20^{\circ}\right)$ and perpendicular $\left(\theta=90^{\circ}\right)$ to the surface, respectively, with a slight preference for the perpendicular one.

Gautam et al.'s SFG experiment of PS on sapphire [17] showed that the phenyl-ring planes at the sapphire interface mostly lay down on the interface and the averaged orientational angle was $20^{\circ}$, which corresponds to the maximum at $\theta=20^{\circ}$ in Figure 6. Meanwhile, Wilson et al.'s experiment [18] showed that the phenyl ring orientation was highly dependent on the characteristics of the substrates. According to their results, a hydrophilic surface, such as UV- 
ozone treated glass, causes the phenyl-ring planes to orient parallel to the surface while a hydrophobic surface induces the perpendicular orientation. In other words, their experiments indicate that when the attractive interaction between the substrate and the phenyl rings is strong, the phenyl-ring planes are oriented parallel to the interface, while when the attractive interaction is weak such that the intramolecular interactions of PS molecules become more important, the phenyl-ring planes tend to orient perpendicular to the surface. From this point of view, it seems that the graphite surface exhibits behavior intermediate between hydrophilic and hydrophobic limits, with both the parallel and the perpendicular orientation of phenyl-ring planes simultaneously observed at the graphite/PS interface. However, the experimental results should be viewed with some caution since recent SFG experiments of Tsuruta et al. showed that the PS chain orientation on quartz substrate was strongly dependent upon the film preparation methods from the polymer solutions [19].

Orientational autocorrelation functions of $\boldsymbol{C}-\boldsymbol{H}$ bonds. Figure 7 shows the orientational autocorrelation functions of all the $\mathrm{C}-\mathrm{H}$ bond vectors in the three different regions as described above. The function is the same as defined in equation 7; however, here $\theta(t)$ is an angle between a $\mathrm{C}-\mathrm{H}$ bond at a designated starting time and after the elapsed time $t$. He et al. [58] showed that such functions followed the modified Kohlrausch-Williams-Watts (mKWW) equation from their MD simulations of PS melt chains and that the average relaxation times from the simulations were in good agreement with the NMR experiments. In this work we find that the orientational autocorrelation function of $\mathrm{C}-\mathrm{H}$ bonds varies depending on their location in the thin film. The autocorrelation functions were thus calculated as follows. First, the $z$ coordinate of the center of each $\mathrm{C}-\mathrm{H}$ vector was recorded at the beginning of the time scan and assigned to one of the three regions, as defined in Figure 6. Next, during the time scan, the new positions were recorded as a function of time and reassigned to the corresponding region. A $\mathrm{C}-\mathrm{H}$ bond contributes to the 
averaged autocorrelation function only if it remains in the same region as assigned at the beginning of the time scan. Therefore, after the elapse of $300 \mathrm{ps}$ the $\mathrm{C}-\mathrm{H}$ bonds that have always remained in the vacuum interface Region III are such a small fraction $(<5 \%)$ of the initial populations that the autocorrelation function in Region III is not plotted beyond $300 \mathrm{ps}$. As shown in Figure 7, the C-H bonds in the graphite interface Region I lose the orientational autocorrelation over a much longer time as compared with those in the middle bulk-like Region II. Moreover, the decay of their orientational autocorrelations in the vacuum interface Region III is much faster than in the middle Region II, especially at short times. For quantitative comparisons, we obtained the segmental relaxation time of $\mathrm{C}-\mathrm{H}$ bonds in each region by fitting the autocorrelation function to the mKWW equation defined as:

$$
P_{m K W W}(t)=a_{l i b} \exp \left(-t / \tau_{l i b}\right)+\left(1-a_{l i b}\right) \exp \left(-\left(t / \tau_{\text {seg }}\right)^{\beta}\right)
$$

where $a_{l i b}$ and $\tau_{l i b}$ represent the amplitude and the relaxation time for the librational motion whereas $\tau_{\text {seg }}$ and $\beta$ are the parameters that describe the segmental relaxations [58]. In the middle Region II, $\tau_{\text {seg }}$ is $\sim 4$ ns and $\beta$ is $\sim 0.45$, which are reasonable values when compared with the results of He et al. for bulk PS melts [58]. In comparison, in the graphite interface Region I $\tau_{\text {seg }}$ is $\sim 80 \mathrm{~ns}$ and $\beta$ is $\sim 0.35$, whereas $\tau_{\text {seg }}$ is $\sim 1 \mathrm{~ns}$ and $\beta$ is $\sim 0.33$ in the vacuum interface Region III.

This result clearly shows that the vacuum interface significantly enhances the segmental mobility of PS chains as compared with the bulk melts, whereas the graphite interface strongly reduces it.

\section{Conclusion}

MD simulations of the PS melt in free-standing thin films and on a graphite surface were performed employing an explicit all-atom force field. The surface tension predicted by simulations is in good agreement with experiments within the limits of current simulation 
methods. In the free interfaces, the aromatic phenyl atoms are preferentially populated in the lowdensity outermost low-density surface followed by a depletion of aromatic phenyl-atom density in the adjacent interfacial layer of ca. $7 \AA$ thickness, whereas the backbone alkyl atoms are concomitantly enriched in this layer. These segment density profiles are in good agreement with the results of recent united-atom simulations of free-standing PS thin films, although the phenyl depletion seems less pronounced for the united-atom models [55]. The phenyl-ring planes in the free-surface region are preferentially oriented perpendicular to the surface while the backbone chain vectors orient parallel to the surface, in agreement with experiments $[17,18,75]$ and previous simulations $[55,75]$. In the graphite interface, the backbone chain vectors are strongly oriented along the graphite surface, whereas the distribution of phenyl ring-normal vectors shows two maxima along the nearly parallel $\left(20^{\circ}\right)$ and perpendicular direction to the surface normal. The segmental mobility of PS melt chains in the graphite interface is strongly diminished due to the interactions between the graphite surface and the aromatic phenyl groups of PS, whereas in the free surface region the segmental mobility is enhanced as compared with the bulk PS melts due to the decreased segment density.

As compared with previous studies of free-standing thin films of PE [40] and PTFE [49], the results of PS films exhibit common features of over-all density profiles, distribution of chain ends, orientation of chain vectors and enhanced chain dynamics at the free-surface region. However, the PS films are quite unique in that they exhibit a nonuniform distribution of chain segments in the interface region by depleting phenyl groups and concomitantly enriching backbone alkyl groups in the interfacial layer adjacent to the low-density free-surface region. Since the abundance of phenyl groups in PS chains provides strong aromatic-aromatic interactions in the melts, the observation of the phenyl-depleted interfacial layer may provide a significant clue to understanding the origin of the decreased $T_{g}$ in thin PS films, most pronounced 
among all the polymers [6,54]. This seems to be supported by the recent simulation study of glass transition in free-standing PS thin films employing a united-atom model that adequately reproduces the key structural characteristics of PS surfaces obtained by explicit-atom simulations [55].

ACKNOWLEDGMENT: This work was supported in part by National Research Foundation of Korea (R01-2008-000-11971-0) and by the Chemistry and Molecular Engineering Program of the Brain Korea 21 Project. The authors sincerely acknowledge the helpful discussions with Wen-li Wu and Christopher L. Soles of NIST concerning the experimental studies of polymer thin films. AVL is grateful to the members of the Frank group and the staff of the chemical engineering department for their hospitality during his sabbatical stay at Stanford University.

\section{References and Notes}

(1) I.C. Sanchez ed., Physics of Polymer Surfaces and Interfaces, Butterworth-Heinemann, Boston, 1992.

(2) J.L. Keddie, R.A.L. Jones, R.A. Cory, Size-dependent depression of the glass transition temperature in polymer films, Europhys. Lett. 27 (1994) 59-64.

(3) J.A. Forrest, K. Dalnoki-Veress, J.R. Stevens, J.R. Dutcher, Effect of free surfaces on the glass transition temperature of thin polymer films, Phys. Rev. Lett. 77 (1996) 2002-2005. 
(4) T. Kajiyama, K. Tanaka, A. Takahara, Surface molecular motion of the monodisperse polystyrene films, Macromolecules 30 (1997), 280-285.

(5) K. Tanaka, A. Takahara, T. Kajiyama, Rheological analysis of surface relaxation process of monodisperse polystyrene films, Macromolecules 33 (2000), 7588-7593.

(6) M.D. Ediger, J.A. Forrest, Dynamics near free surfaces and the glass transition in thin polymer films: a view to the future, Macromolecules 47 (2014) 471-478.

(7) W.E. Wallace, J.H. van Zanten, W.L. Wu, Influence of an impenetrable interface on a polymer glass-transition temperature, Phys. Rev. E 52 (1995) R3329-R3332.

(8) G.B. DeMaggio, W.E. Frieze, D.W. Gidley, M. Zhu, H.A. Hristov, A.F. Yee, Interface and surface effects on the glass transition in thin polystyrene films, Phys. Rev. Lett. 78 (1997) $1524-1527$.

(9) J.A. Forrest, K. Dalnoki-Veress, J.R. Dutcher, Interface and chain confinement effects on the glass transition temperature of thin polymer films, Phys. Rev. E 56 (1997) 5705-5714.

(10) J.R. Potts, D.R. Dryer, C.W. Bielawski, R.S. Rouoff, Graphene-based polymer nanocomposites, Polymer 53 (2011) 5-25.

(11) M. Birkholz, Thin Film Aalysis by X-ray Scattering, Wiley-VCH, Weinheim, 2006.

(12) J. Stöhr, NEXAFS Spectroscopy, Springer-Verlag, Berlin, 1992.

(13) D. Briggs, Surface Analysis of Polymers by XPS and Static SIMS, Cambridge University Press, Cambridge, 1998. 
(14) B.D. Ratner, V.V. Tsukruk, Scanning Probe Microscopy of Polymers, ACS Symposium Series 694, American Chemical Society, Washington DC, 1998.

(15) Z. Chen, Y.R. Shen, G.A. Somorjai, Studies of polymer surfaces by sum frequency generation vibrational spectroscopy, Annu. Rev. Phys. Chem. 53 (2002) 437-465.

(16) Z. Chen, Understanding surfaces and buried interfaces of polymer materials at the molecular level using sum frequency generation vibrational spectroscopy, Polym. Int. 56 (2007) $577-587$.

(17) K.S. Gautam, A.D. Schwab, A. Dhinojwala, D. Zhang, S.M. Dougal, M.S. Yeganeh, Molecular structure of polystyrene at air/polymer and solid/polymer interfaces, Phys. Rev. Lett. 85 (2000) 3854-3857.

(18) P.T. Wilson, L.J. Richter, W.E. Wallace, K.A. Briggman, J.C. Stephenson, Correlation of molecular orientation with adhesion at polystyrene/solid interfaces, Chem. Phys. Lett. 363 (2002) 161-168.

(19) H. Tsuruta, Y. Fujii, N. Kai, H. Kataoka, T. Ishizone, M. Doi, H. Morita, K. Tanaka, Local conformation and relaxation of polystyrene at substrate interface, Macromolecules 45 (2012) $4643-4649$.

(20) A. Rao, H. Rangwalla, V. Varshney, A. Dhinojwala, Structure of poly(methyl methacrylate) chains adsorbed on sapphire probed using infrared-visible sum frequency generation spectroscopy, Langmuir 20 (2004) 7183-7188.

(21) K.S. Gautam, A. Dhinojwala, Melting at alkyl side chain comb polymer interfaces, Phys. Rev. Lett. 88 (2002) 14550-1-4. 
(22) J. Kim, G.A. Somorjai, Molecular packing of lysozyme, fibrinogen, and bovine serum albumin on hydrophilic and hydrophobic surfaces studied by infrared-visible sum frequency generation and fluorescence microscopy, J. Am. Chem. Soc. 125 (2003) 3150-3158.

(23) X. Chen, M.L. Clarke, J. Wang, Z. Chen, Sum frequency generation vibrational spectroscopy studies of molecular conformation and orientation of biological molecules at interfaces, Int. J. Mod. Phys. B 19 (2005) 691-713.

(24) C.L. Soles, J.F. Douglas, W.-L. Wu, R.M. Dimeo, Incoherent neutron scattering and the dynamics of confined polycarbonate films, Phys. Rev. Lett. 88 (2002) 037401-1-4.

(25) C.L. Soles, J.F. Douglas, E.K. Lin, J.L. Lenhart, R.L. Jones, W.-L. Wu, D.L. Goldfarb, M. Angelopoulos, Incoherent neutron scattering and the dynamics of thin film photoresist polymers, J. Appl. Phys. 93 (2003) 1978-1986.

(26) C.L. Soles, J.F. Douglas, W.-L. Wu, R.M. Dimeo, Incoherent neutron scattering as a probe of the dynamics in molecularly thin polymer films, Macromolecules 36 (2003) 373-379.

(27) R. Inoue, T. Kanaya, K. Nishida, I. Tsukushi, J. Taylor, S. Levett, B.J. Gabrys, Dynamic anisotropy and heterogeneity of polystyrene thin films as studied by inelastic neutron scattering, Eur. Phys. J. E 24 (2007) 55-60.

(28) P. Dorucker, W.L. Mattice, Mobility of the surface and interior of thin films composed of amorphous polyethylene, Macromolecules 32 (1999) 194-198.

(29) K.F. Mansfield, D.N. Theodorou, Interfacial structure and dynamics of macromolecular liquids: a Monte Carlo simulation approach, Macromolecules 22 (1989) 3143-3152. 
(30) G.D. Smith, D.Y. Yoon, R.L. Jaffe, Conformations of polymer melts between parallel surfaces: comparison of the Scheutjens-Fleer lattice theory with Monte Carlo simulations, Macromolecules 25 (1992) 7011-7017.

(31) I.A. Bitsanis, G. ten Brinke, A lattice Monte Carlo study of long chain conformations at solid-polymer melt interfaces, J. Chem. Phys. 99 (1993) 3100-3111.

(32) R. Dickman, C.K. Hall, High density Monte Carlo simulations of chain molecules: bulk equation of state and density profile near walls, J. Chem. Phys. 89 (1988) 3168-3174.

(33) S.K. Kumar, M. Vacatello, D.Y. Yoon, Off-lattice Monte Carlo simulations of polymer melts confined between two plates, J. Chem. Phys. 89 (1988) 5206-5215.

(34) A. Yethiraj, Monte Carlo simulation of confined semiflexible polymer melts, J. Chem. Phys. 101 (1994) 2489-2497.

(35) J. Baschnagel, H. Meyer, F. Varnik, S. Metzger, M. Aichele, M. Müller, K. Binder, Computer simulations of polymers close to solid interfaces: some selected topics, Interface. Science. 11 (2003) 159-173.

(36) I. Bitsanis, G. Hadziioannou, Molecular dynamics simulations of the structure and dynamics of confined polymer melts, J. Chem. Phys. 92 (1990) 3827-3847.

(37) L. Delle Site, C.F. Abrams, A. Alavi, K. Kremer, Polymers near metal surfaces: selective adsorption and global conformations, Phys. Rev. Lett. 89 (2002) 156103-1-4.

(38) X. Li, Y. Hu, H. Wang, Modeling of lubricant spreading on a solid substrate, J. Appl. Phys. 99 (2006) 024905-1-5. 
(39) P.A. Thompson, G.A. Grest, M.O. Robinson, Phase transitions and universal dynamics in confined films, Phys. Rev. Lett. 68 (1992) 3448-3451.

(40) J. Chang, J. Han, L. Yang, R.L. Jaffe, D.Y. Yoon, Structure and properties of polymethylene melt surfaces from molecular dynamics simulations, J. Chem. Phys. 115 (2001) $2831-2840$.

(41) K.F. Mansfield, D.N. Theodorou, Atomistic simulation of a glassy polymer/graphite interface, Macromolecules 24 (1991) 4295-4309.

(41) K.F. Mansfield, D.N. Theodorou, Molecular dynamics simulation of a glassy polymer surface, Macromolecules 24 (1991) 6283-6294.

(43) J.G. Harris, Liquid-vapor interfaces of alkane oligomers. structure and thermodynamics from molecular dynamics simulations of chemically realistic models, J. Phys. Chem. 96 (1992) 5077-5086.

(44) T. Hapke, G. Pätzold, D.W. Heermann, Surface tension of amorphous polymer films, J. Chem. Phys. 109 (1998) 10075-10081.

(45) A. Hariharan, J.G. Harris, Structure and thermodynamics of the liquid-vapor interface

of fluorocarbons and semifluorinated alkane diblocks: a molecular dynamics study, J. Chem. Phys. 101 (1994) 4156-4165.

(46) T.C. Clancy, J.H. Jang, A. Dhinojwala, W.L. Mattice, Orientation of phenyl rings and methylene bisectors at the free surface of atactic polystyrene, J. Phys. Chem. B 105 (2001) 11493-11497. 
(47) K.C. Daoulas, V.A. Harmandaris, V.G. Mavrantzas, Detailed atomistic simulation of a polymer melt/solid interface: structure, density, and conformation of a thin film of polyethylene melt adsorbed on graphite, Macromolecules 38 (2005) 5780-5795.

(48) D. Hudzinskyy, A.V. Lyulin, A.R.C. Baljon, N.K. Balabaev, M.A.J. Michels, Effects of strong confinement on the glass-transition temperature in simulated atactic polystyrene films, Macromolecules 44 (2011) 2299-2310.

(49) S. Lee, J. Chang, R.L. Jaffe, D.Y. Yoon, Surface and thin film characteristics of poly(tetrafluoroethylene) melts from molecular dynamics simulations, Macromolecules 40 (2007) $7407-7412$.

(50) O. Borodin, G.D. Smith, R. Bandyopadhyaya, O. Byutner, molecular dynamics study of the influence of solid interfaces on poly(ethylene oxide) structure and dynamics, Macromolecules 36 (2003) 7873-7883.

(51) K.S. Khare, R. Khare, Effect of carbon nanotube dispersion on glass transition in crosslinked epoxy-carbon nanotube nanocomposites: role of interfacial interactions, J. Phys. Chem. B 117 (2013) 7444-7454.

(52) M. Solar, W. Paul, Dielectric $\alpha$-relaxation of 1,4-polybutadiene confined between graphite walls, Euro. Phys. J. E. 39 (2015) 37-48.

(53) G.D. Smith, C. Ayyagari, R.L. Jaffe, M. Pekny, A. Bernarbo, Conformations of 2,4diphenylpentane: a quantum chemistry and gas-phase molecular dynamics simulation study, J. Phys. Chem. A 102 (1998) 4694-4702.

(54) K. Paeng, M.D. Ediger, Molecular motion in free-standing thin films of poly(methyl 
methacrylate), poly(4-tert-butylstyrene), poly( $\alpha$-methylstyrene), and poly(2-vinylpyridine), Macromolecules 44 (2011) 7034-7042.

(55) A.V. Lyulin, N.K. Balabaev, A.R.C. Baljon, G. Mendoza, C.W. Frank, D.Y. Yoon, Interfacial and topological effects on the glass transition in free-standing polystyrene films, J. Chem. Phys. in press.

(56) C. Ayyagari, D. Bedrov, G.D. Smith, Structure of atactic polystyrene: a molecular dynamics simulation study, Macromolecules 33 (2000) 6194-6199.

(57) H. Höcker, G.J. Blake, P.J. Flory, P. J. Equation-of-state parameters for polystyrene, Trans. Faraday Soc. 67 (1971) 2251-2257.

(58) Y. He, T.R. Lutz, M.D. Ediger, C. Ayyagari, D. Bedrov, G.D. Smith, NMR experiments and molecular dynamics simulations of the segmental dynamics of polystyrene, Macromolecules 37 (2004) 5032-5039.

(59) W.A. Steele, The physical interactions of gases with crystalline solids. I. gas-solid energies and properties of isolated adsorbed atoms, Surf. Sci. 36 (1973) 317-352.

(60) W.L. Jorgensen, D.S. Maxwell, J. Tirado-Rives, Development and testing of the OPLS allatom force field on conformational energetics and properties of organic liquids, J. Am. Chem. Soc. 118 (1996) 11225-11236.

(61) G.J. Martyna, M.L. Klein, M. Tuckerman, Nosé-Hoover chains: the canonical ensemble via continuous dynamics, J. Chem. Phys. 97 (1992) 2635-2643. 
(62) G.J. Martyna, D.J. Tobias, M.L. Klein, Constant pressure molecular dynamics algorithms, J. Chem. Phys. 101 (1994) 4177-4189.

(63) G.J Martyna, M.E. Tuckerman, D.J. Tobias, M.L. Klein, Explicit reversible integrators for extended systems dynamics, Mol. Phys. 87 (1996) 1117-1157.

(64) F.S. Csajka, C. Seidel, Strongly charged polyelectrolyte brushes: a molecular dynamics

Study, Macromolecules 33 (2000) 2728-2739.

(65) J. Lekner, Summation of Coulomb fields in computer-simulated disordered systems, Physica A 176 (1991) 485-498.

(66) R. Sperb, Extension and simple proof of Lekner's summation formula for Coulomb forces Mol. Simul. 13 (1994) 189-193.

(67) M. Tuckerman, B.J. Berne, G.J. Martyna, Reversible multiple time scale molecular dynamics, J. Chem. Phys. 97 (1992) 1990-2001.

(68) M.P. Allen, D.J. Tildesley, Computer Simulation of Liquids, Clarendon, Oxford, 1990.

(69) J.G. Kirkwood, F.P. Buff, The statistical mechanical theory of surface tension, J. Chem. Phys. 17 (1949) 338-343.

(70) J.S. Rowlinson, B. Widom, Molecular Theory of Capillarity, Clarendon, Oxford, 1982.

(71) J. Alejandre, D.J. Tildesley, G.A. Chapela, Molecular dynamics simulation of the orthobaric densities and surface tension of water, J. Chem. Phys. 102 (1995) 4574-4583.

(72) M. Mecke, J. Winkelmann, J. Fisher, Molecular dynamics simulation of the liquid-vapor interface: the Lennard-Jones fluid, J. Chem. Phys. 107 (1997) 9264-9270. 
(73) S. Wu, Surface and interfacial tensions of polymer melts. II. poly(methyl methacrylate), poly(n-butyl methacrylate) and polystyrene, J. Phys. Chem. 74 (1970) 632-638.

(74) J.A. Barker, Surface tension and atomic interactions in simple liquids, Mol. Phys. 80 (1993) 815-820.

(75) J.L. Lenhart, D.A. Fischer, T.L. Chantawansri, J.W. Andzelm, Surface orientation of polystyrene based polymers: steric effects from pendant groups on the phenyl ring, Langmuir 28 (2012) 15713-15724. 


\section{Figure Captions}

Figure 1. Simulation scheme to prepare thin films: (a) equilibrium bulk phase with periodic boundary conditions in 3-dimensions, (b) elongation in the film thickness direction to form freestanding films with periodic boundary conditions in 3-dimension, and (c) introduction of a semiinfinite graphite substrate with periodic boundary conditions in 2-dimension; an enlarged section with the phenyl carbons in green and backbone carbons in red are shown.

Figure 2. Segment density profiles of free-standing PS melt film along the $z$-axis normal to the film surface at $450 \mathrm{~K} ; z=0$ corresponds to the film center. The black solid line is for all atoms, the red dashed line is for phenyl atoms and the blue dotted line is for backbone atoms. For comparison, the densities of phenyl and backbone atoms are scaled to the overall density.

Figure 3. Segment orientational order parameters of designated vectors of free-standing PS melt film at $450 \mathrm{~K}$. (a) Definition of vectors involved in the orientation designation; vector 1 is the normal vector to the film surface, vector 2 is the backbone chain vector and vector 3 is the phenyl-ring normal vector. (b) The segment order parameters in a free-standing PS thin film with respect to the surface normal; the red dashed line is for the phenyl-ring normal and the blue dotted line is for the backbone chain vector. The black solid line is the density profile of all atoms, replotted from Figure 2.

Figure 4. Segment density profiles of PS melt film on graphite substrate along the $z$-axis normal to the film surface at $450 \mathrm{~K} ; z=0$ corresponds to the top of the graphite. (a) The designation of each line is the same as that in Figure 2. (b) Scaled-up plot of density profile of each carbon in a monomer unit at the graphite interface. The density of each species is normalized to that of all atoms. 
Figure 5. (a) Orientational order parameter of designated segment vectors of PS melt film on graphite at $450 \mathrm{~K}$. The designation of each line is the same as that in Figure 3(a) and the solid line is the all-atom density profile, replotted from Figure 4(a). (b) Schematics of PS segments with nearly parallel (left) and perpendicular (right) orientation of the phenyl-ring plane with respect to the graphite surface.

Figure 6. Top: designation of three regions in PS films on graphite as graphite interface (I), bulk (II) and vacuum interface (III). Bottom: distribution of phenyl-ring normal vectors with respect to the surface normal in three different regions at $450 \mathrm{~K}$.

Figure 7. Orientational autocorrelation functions of all the $\mathrm{C}-\mathrm{H}$ vectors at $450 \mathrm{~K}$ in three different regions of PS melt films on graphite. The regions are defined in Figure 6. The red solid lines are fits to the mKWW equation. 


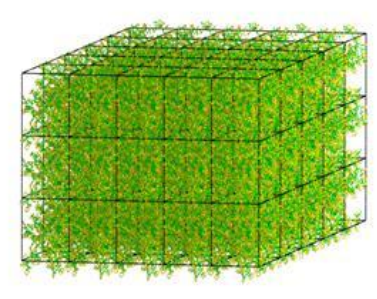

(a)

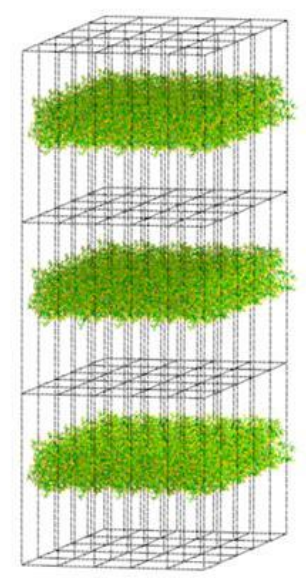

(b)
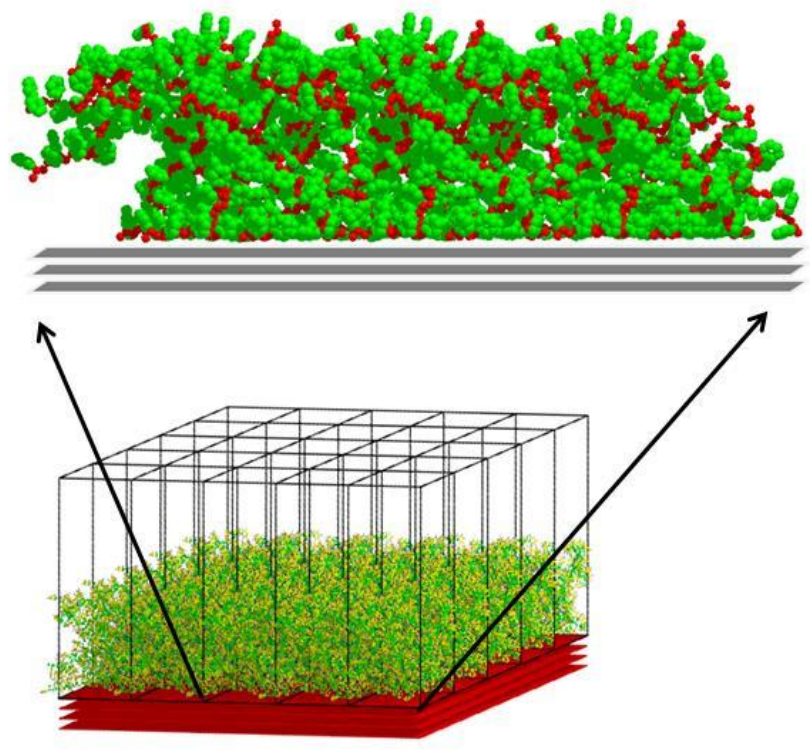

(c)

Figure 1 


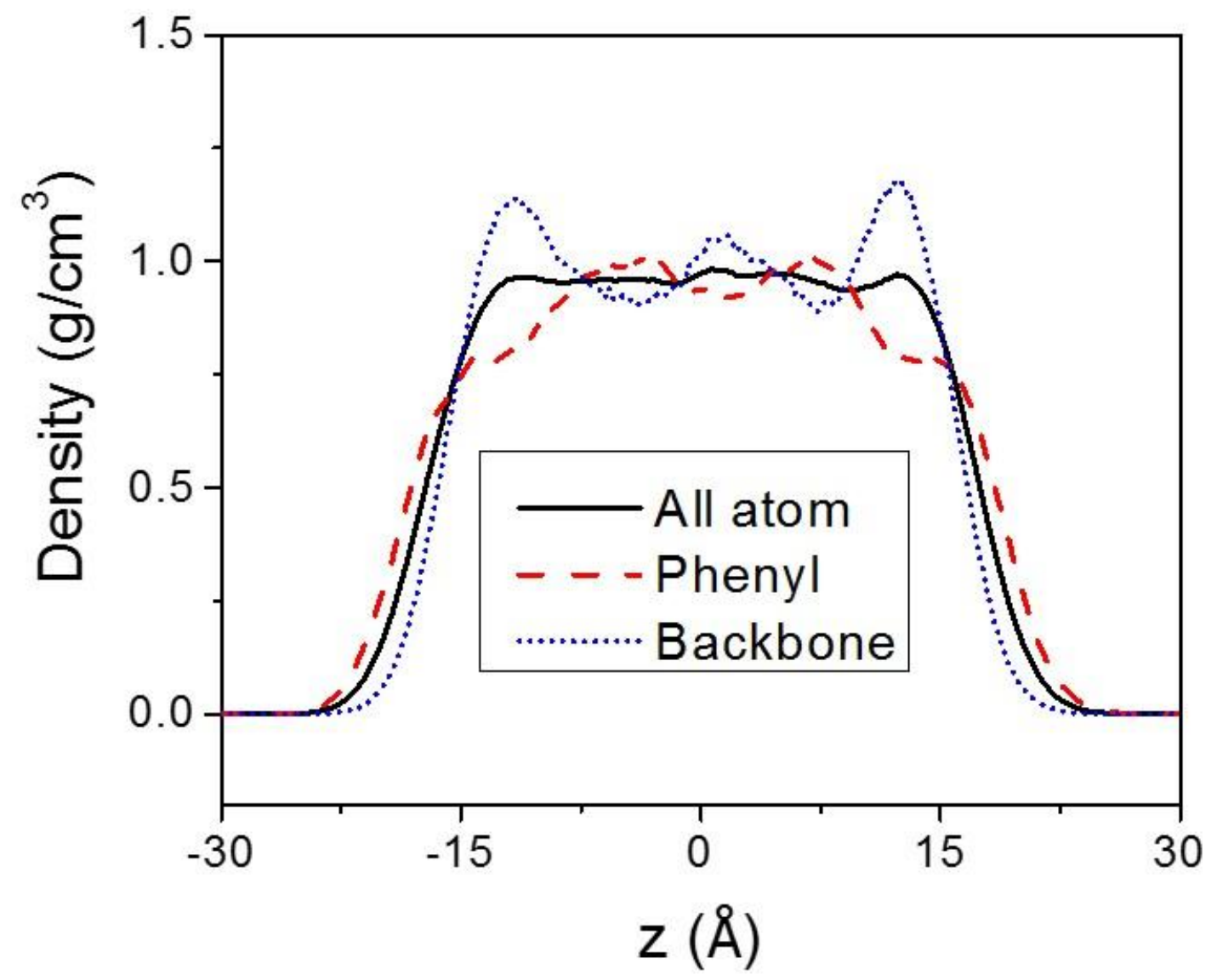

Figure 2 
(a)

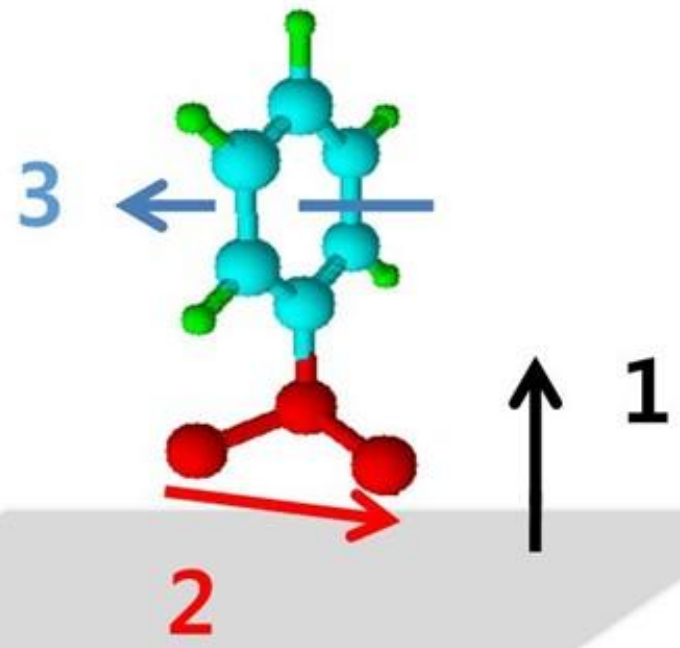

I

(b)

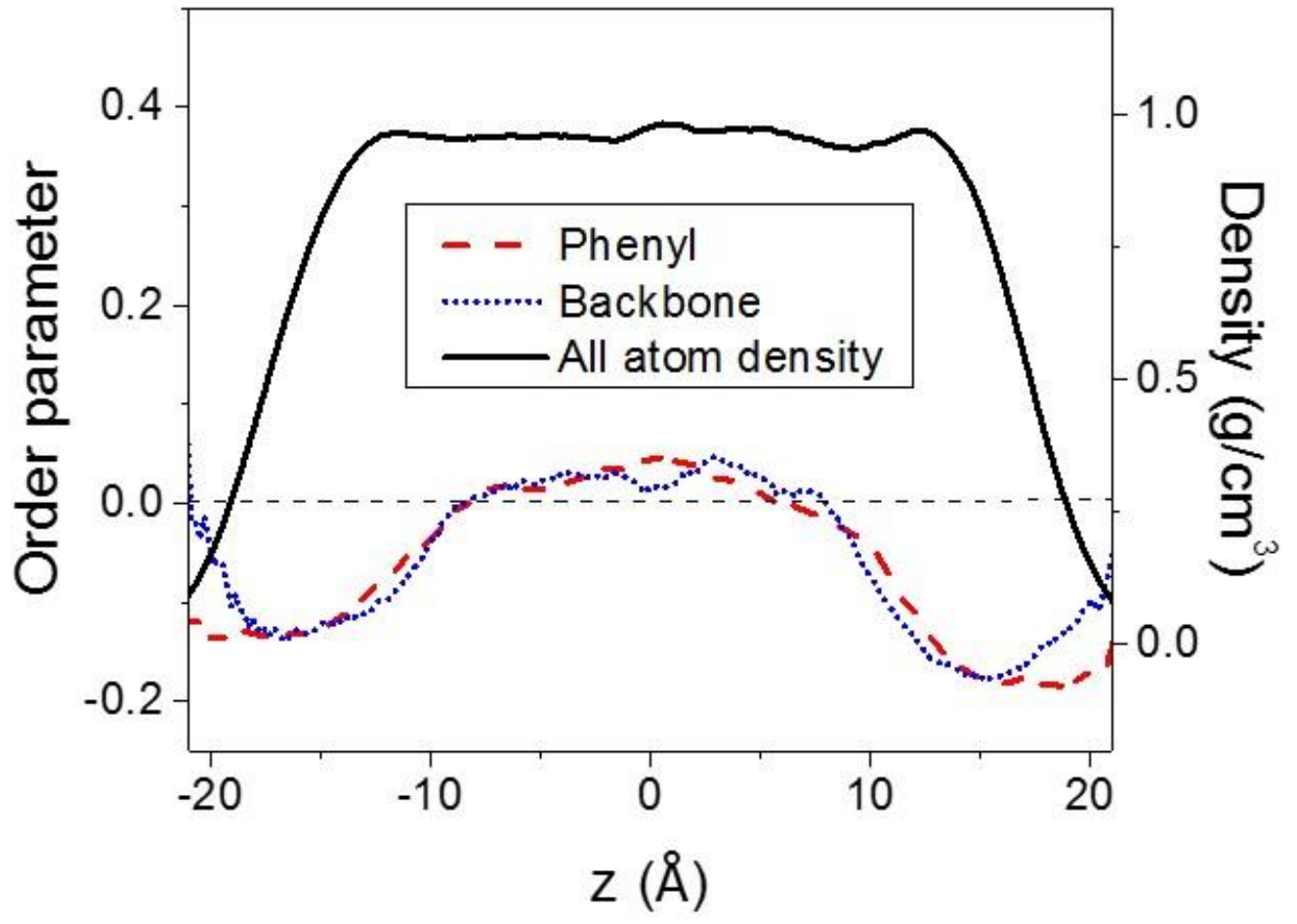

Figure 3. 

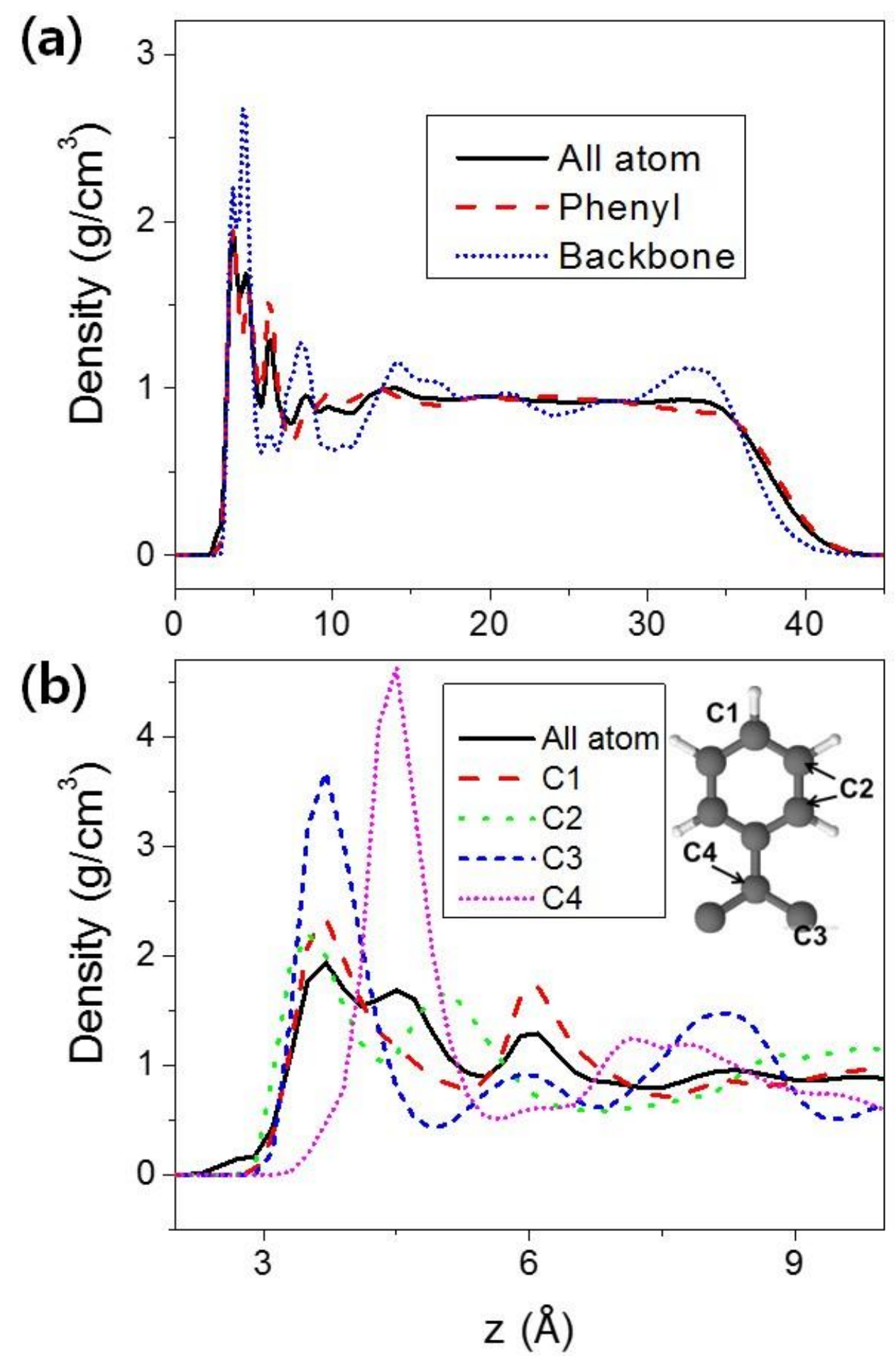

Figure 4. 


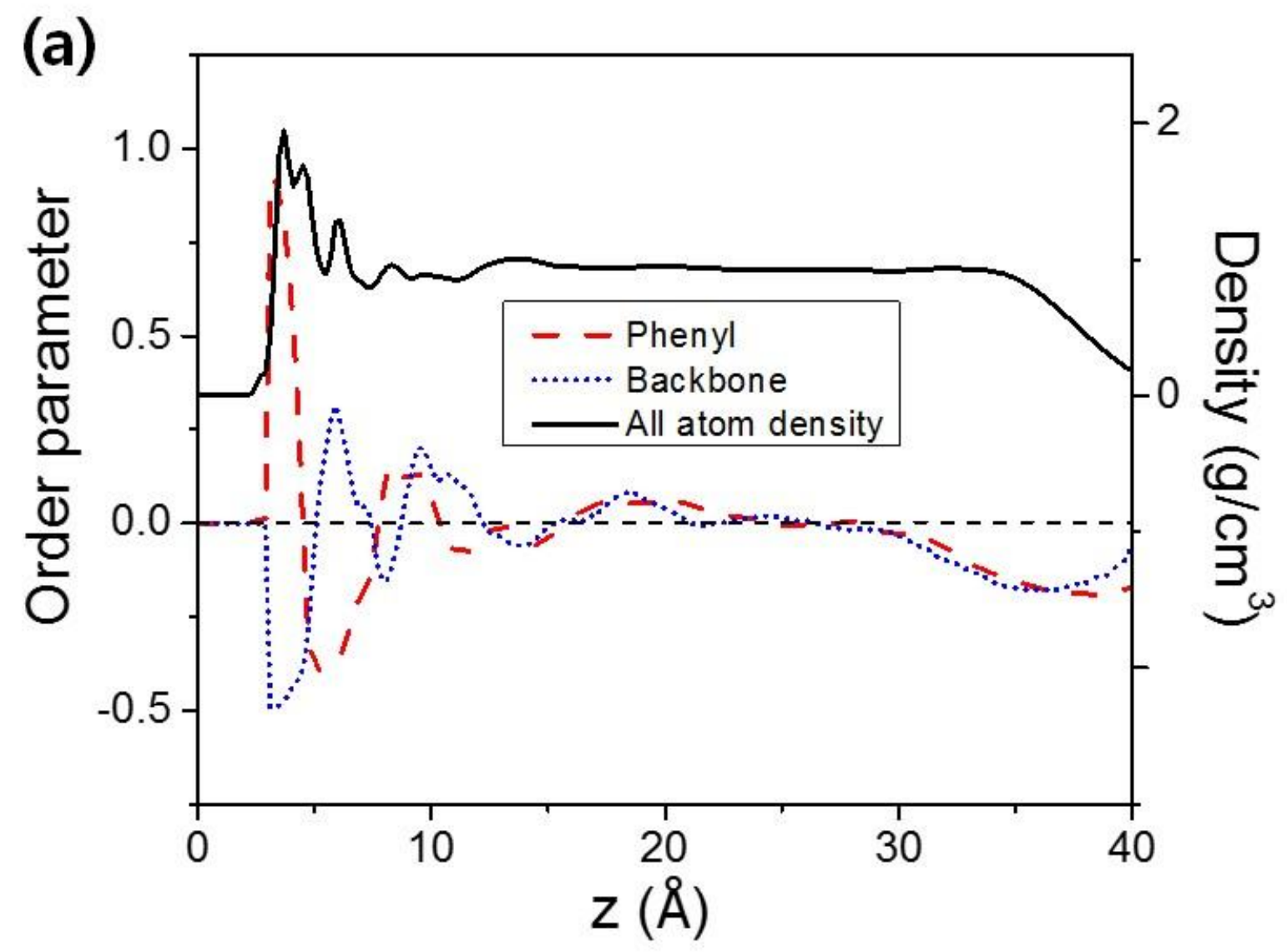

(b)

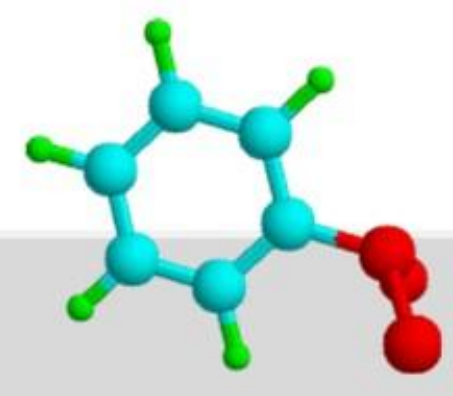

Figure 5. 

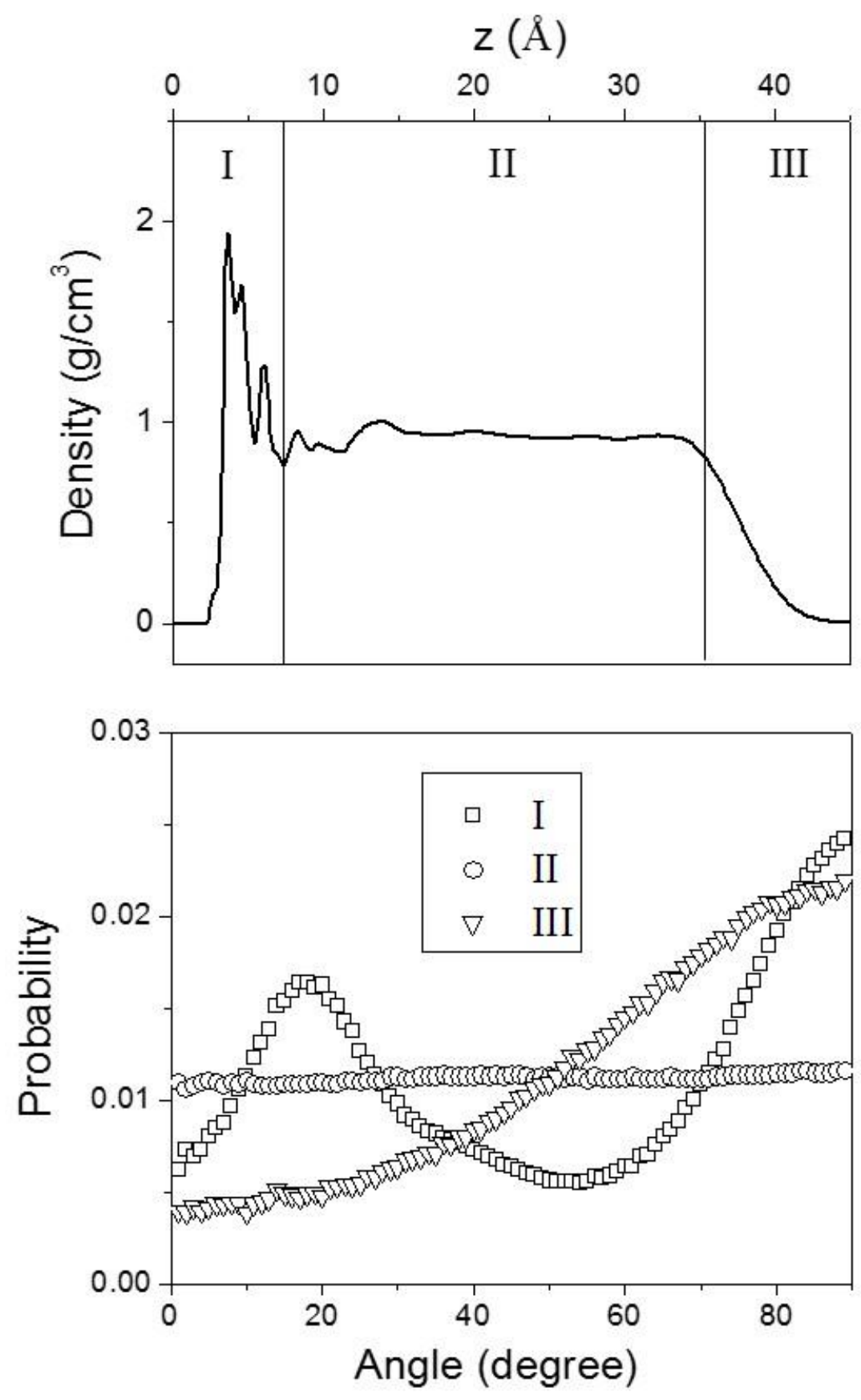

Figure 6. 


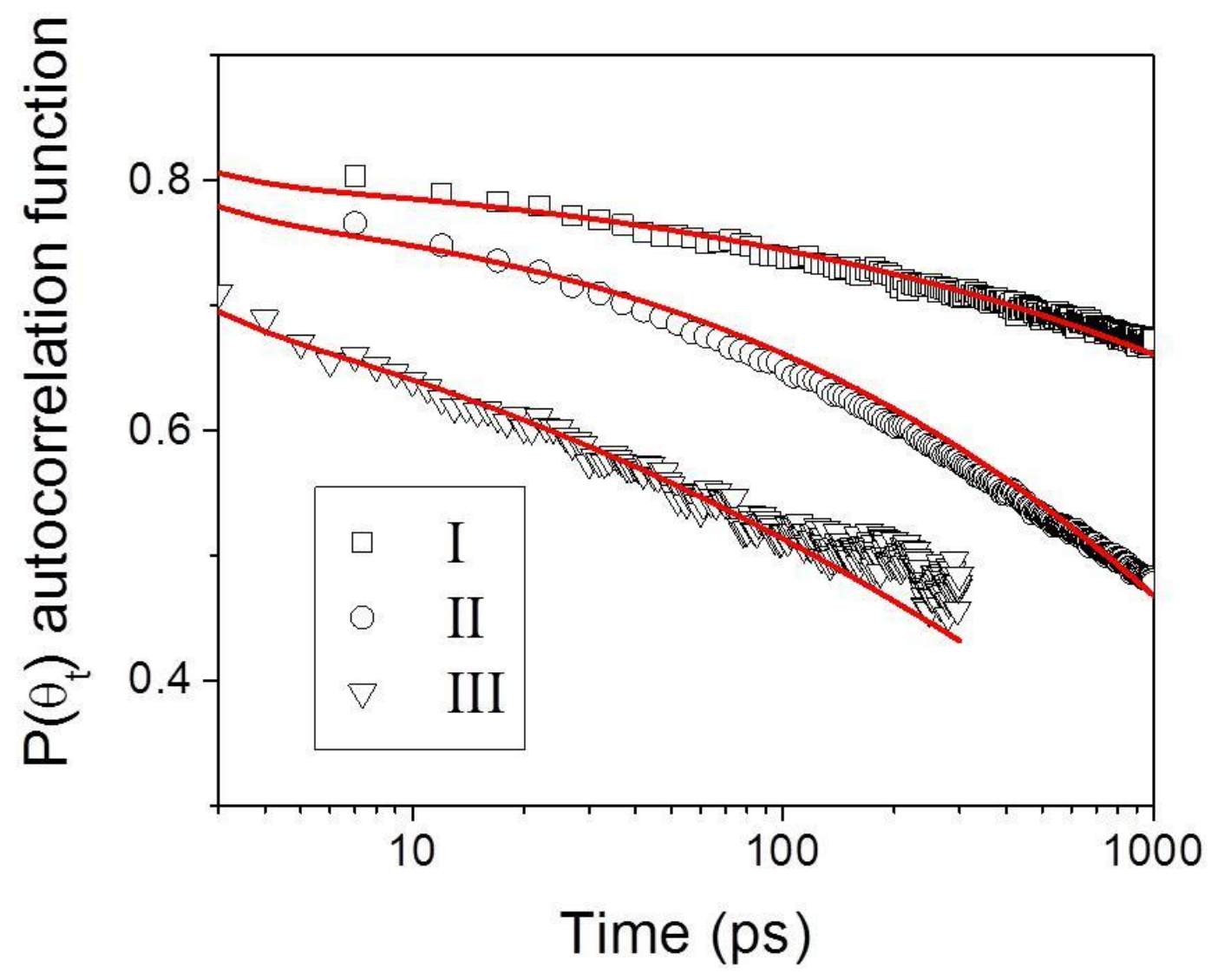

Figure 7. 
Graphical Abstract
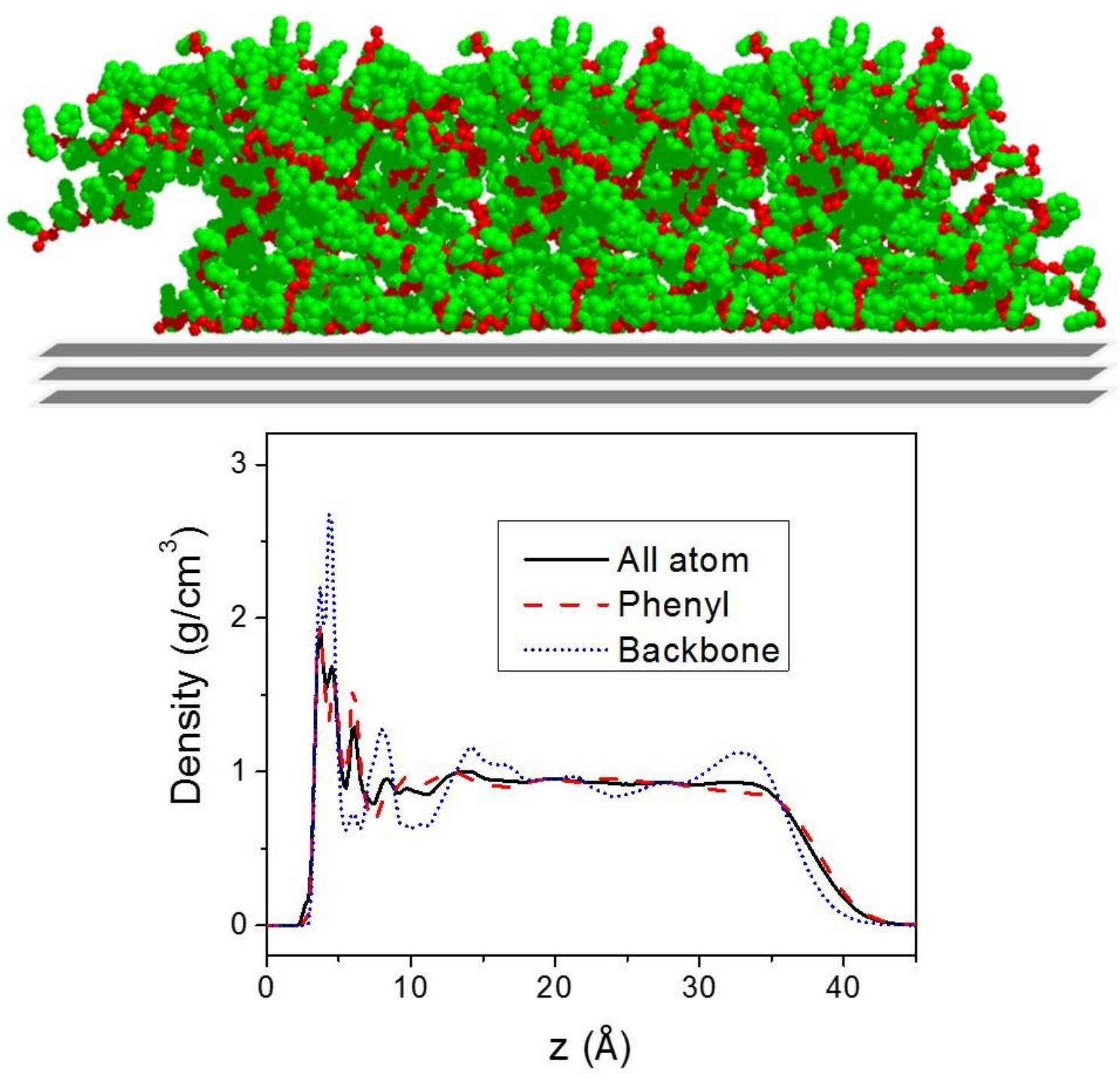


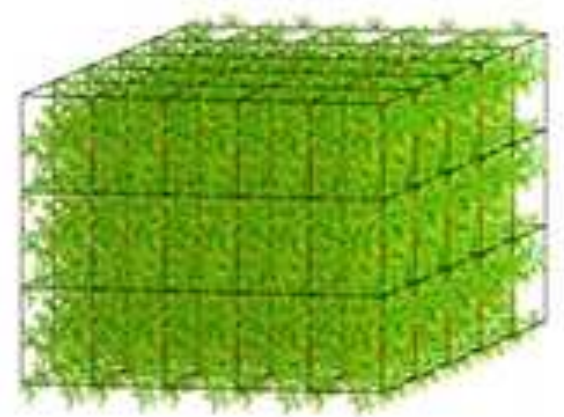

(a)

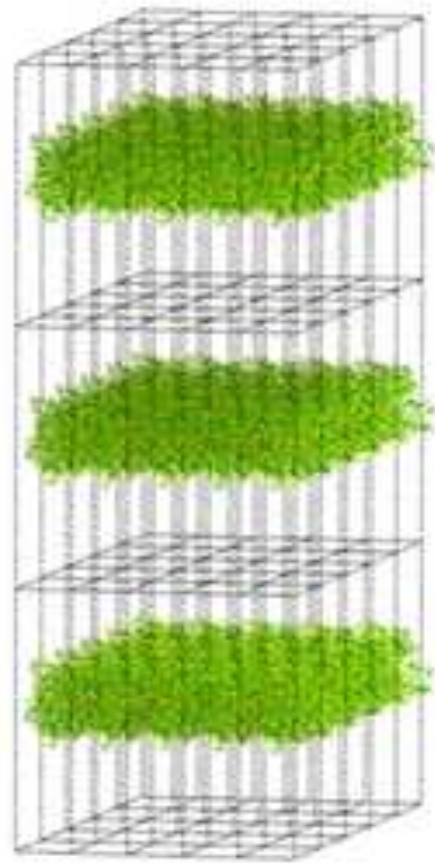

(b)
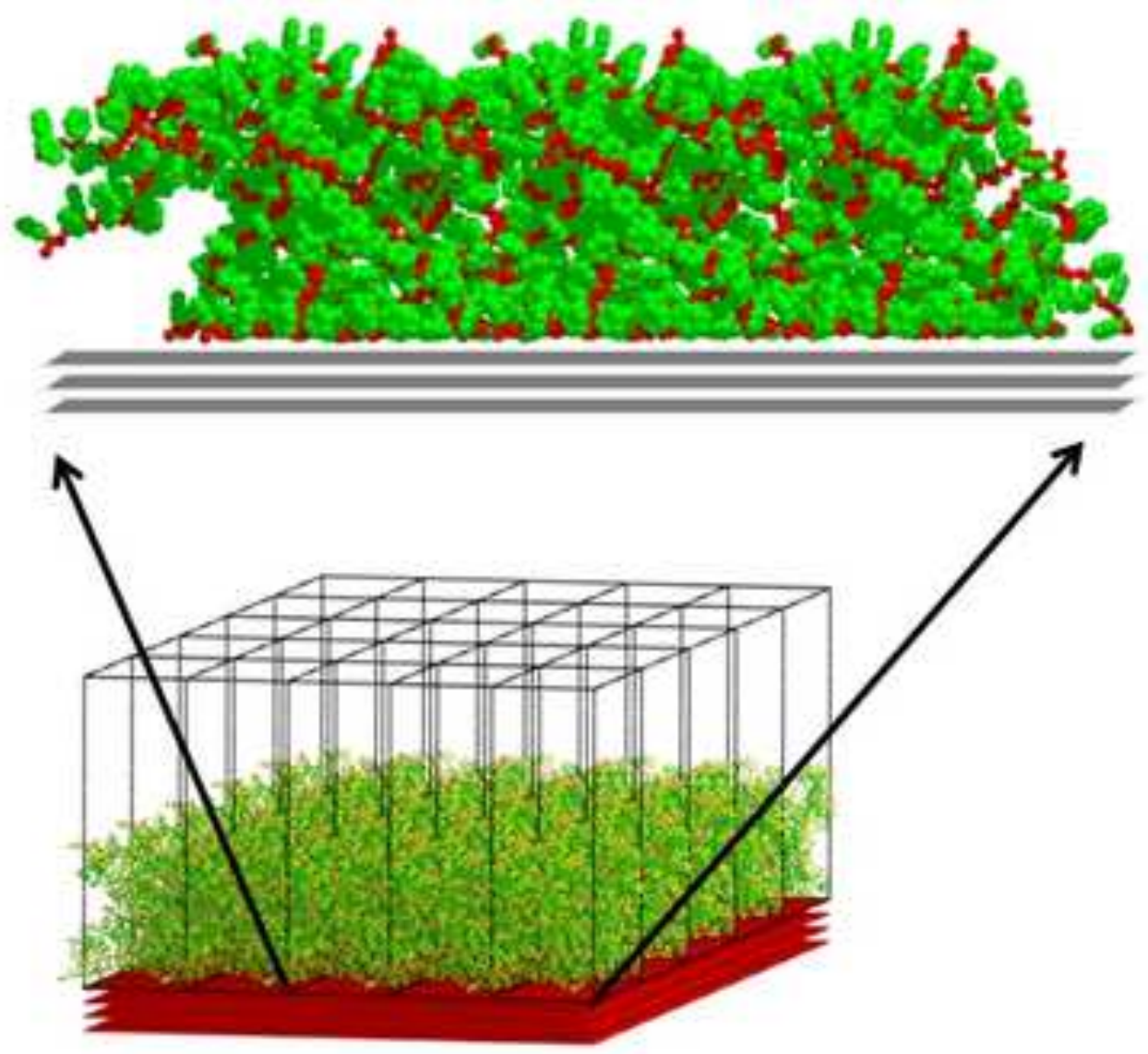

(c) 


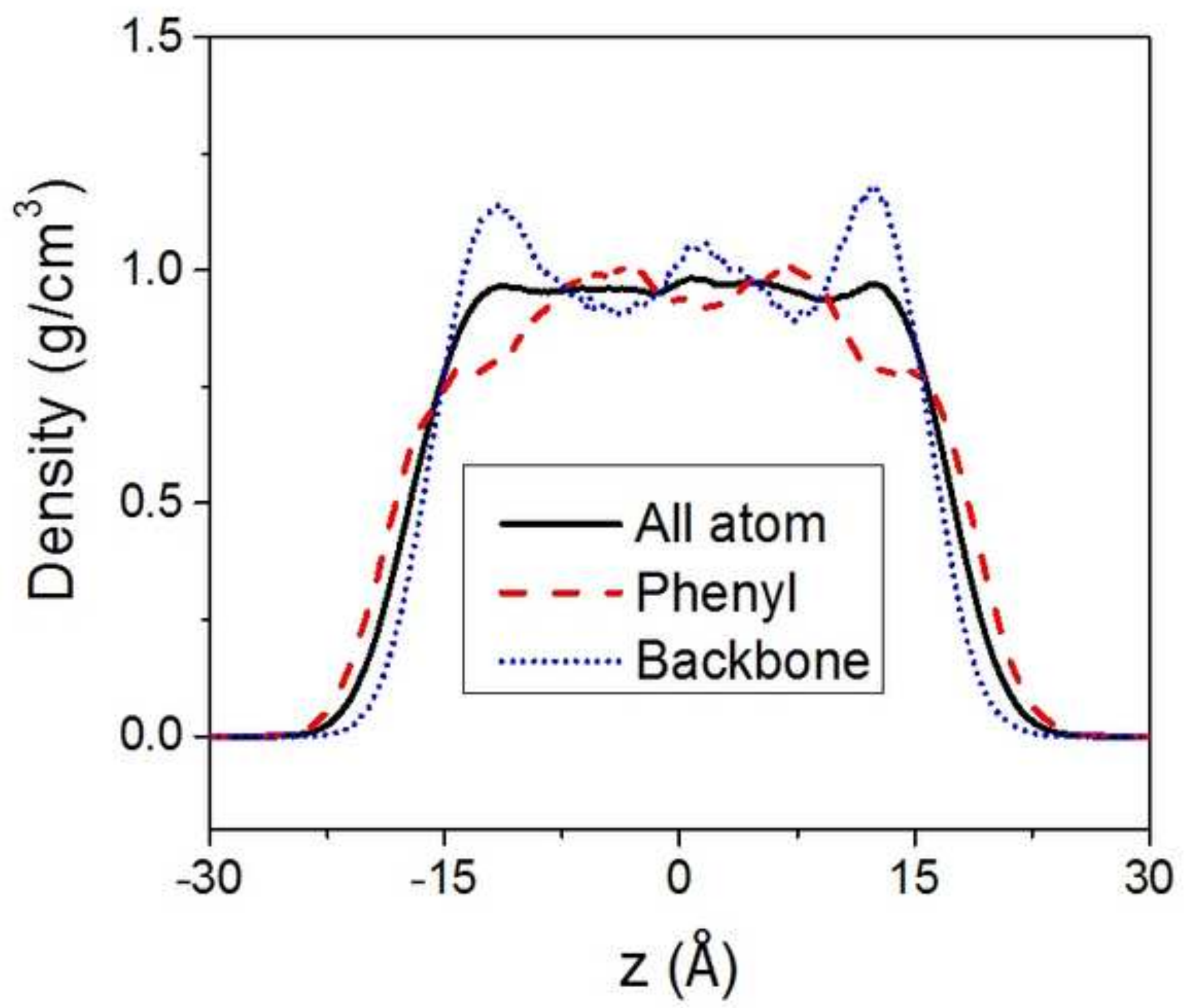


Figure 3

(a)

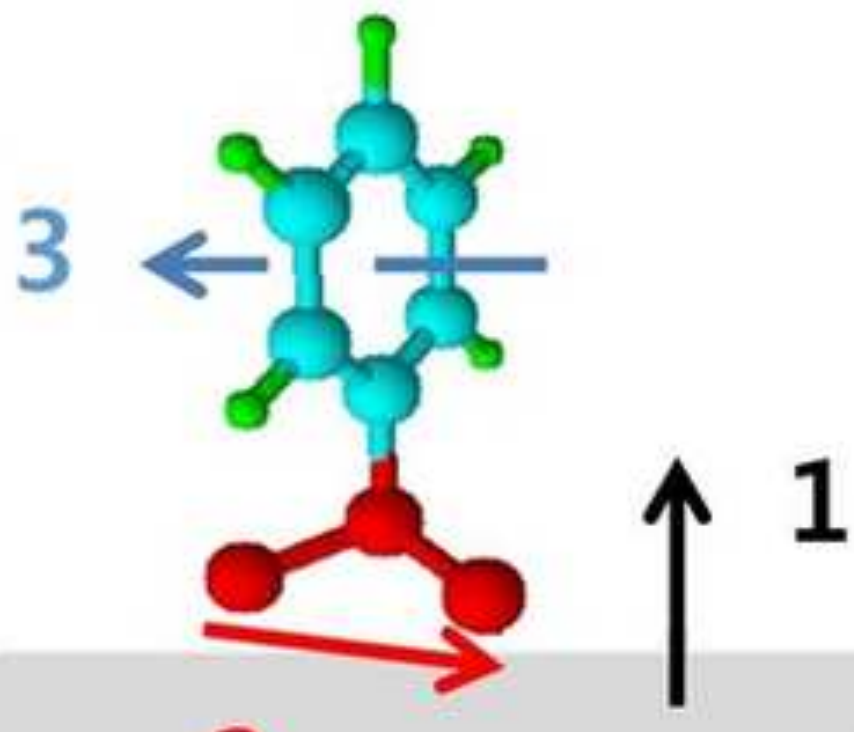

2

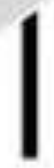

(b)

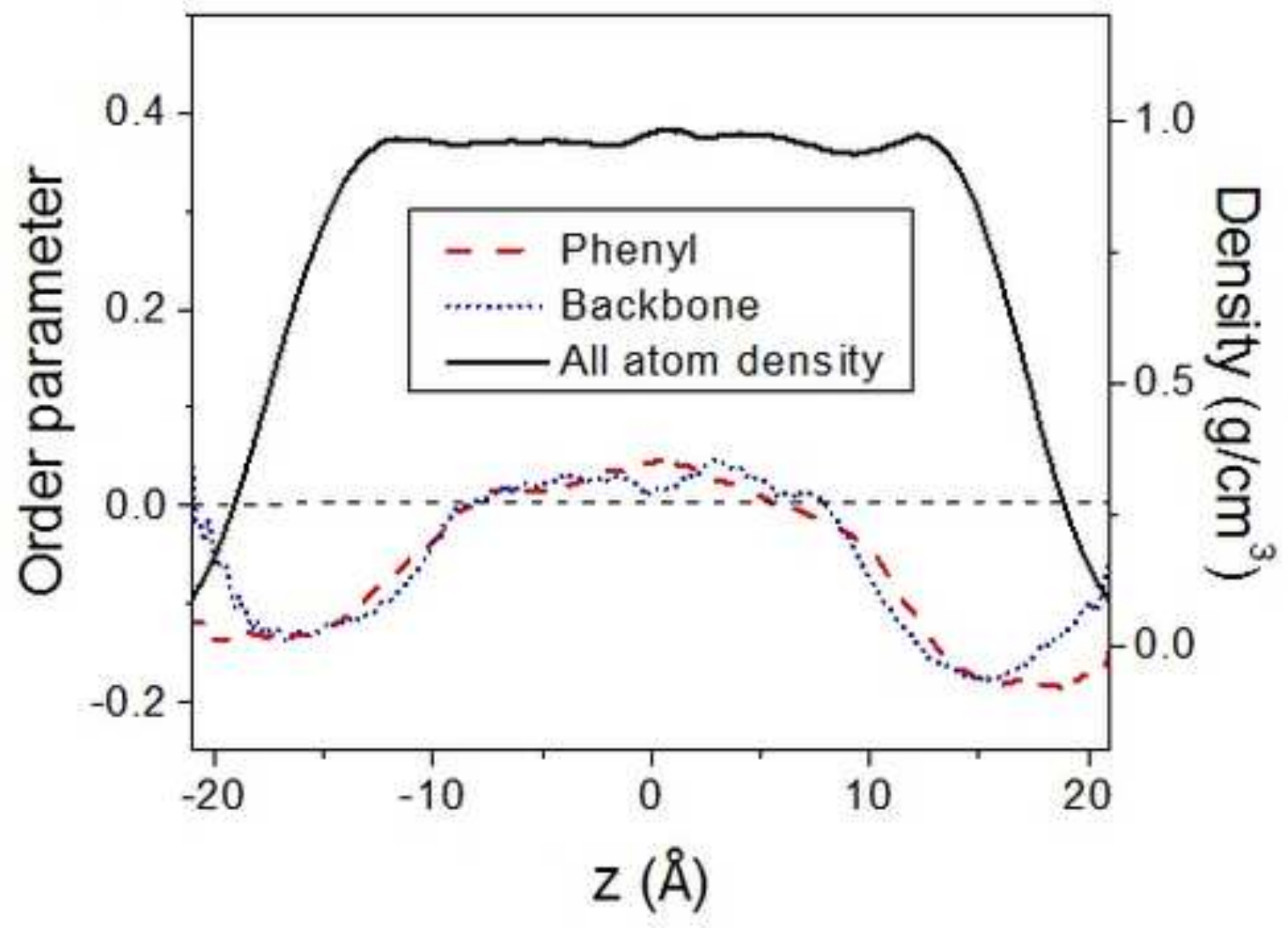


Figure 4
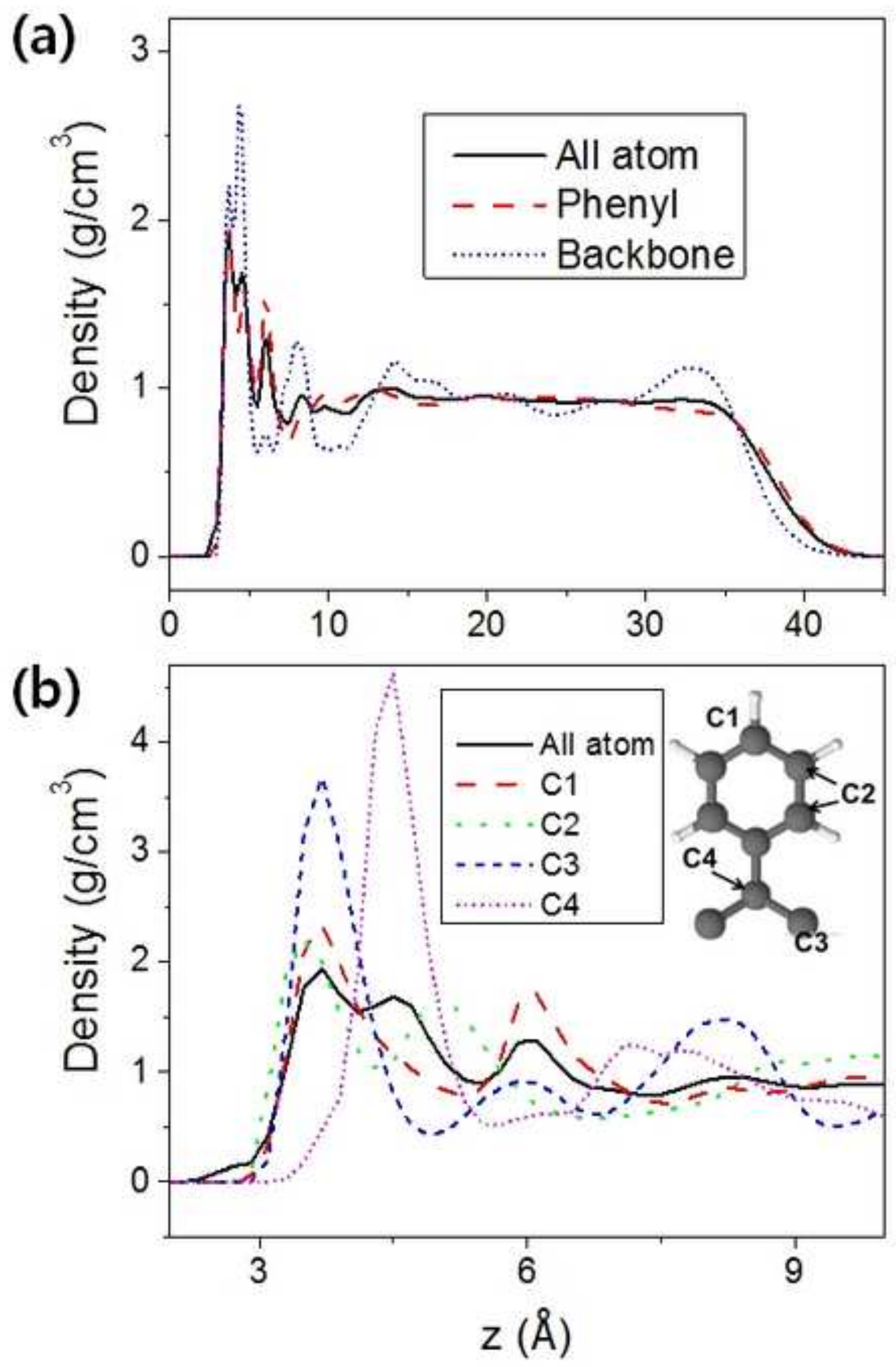


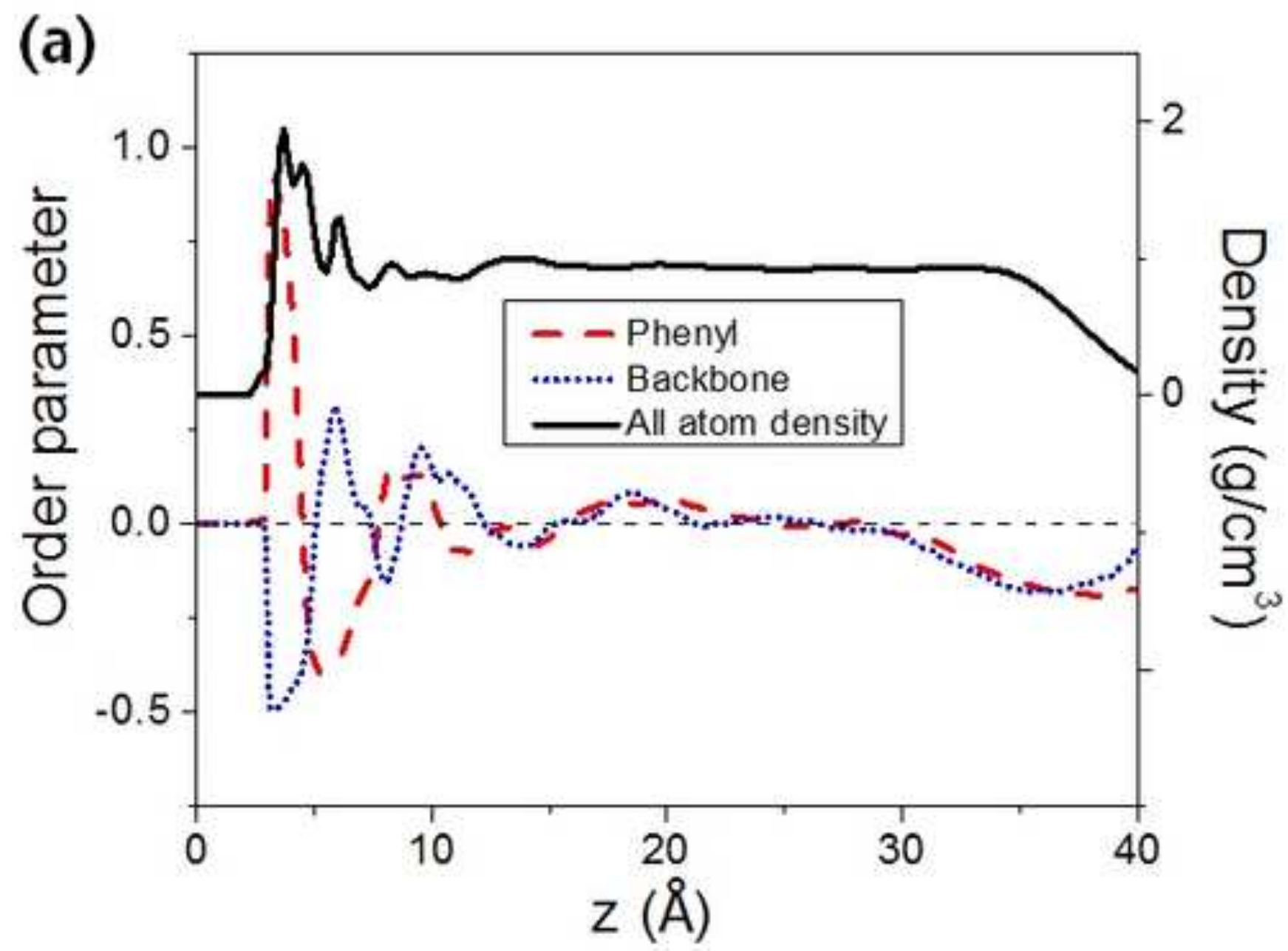

(b)

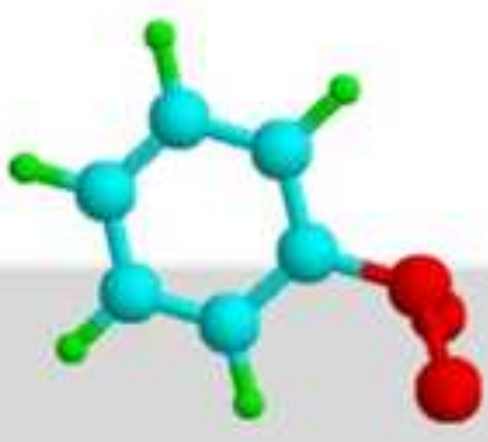



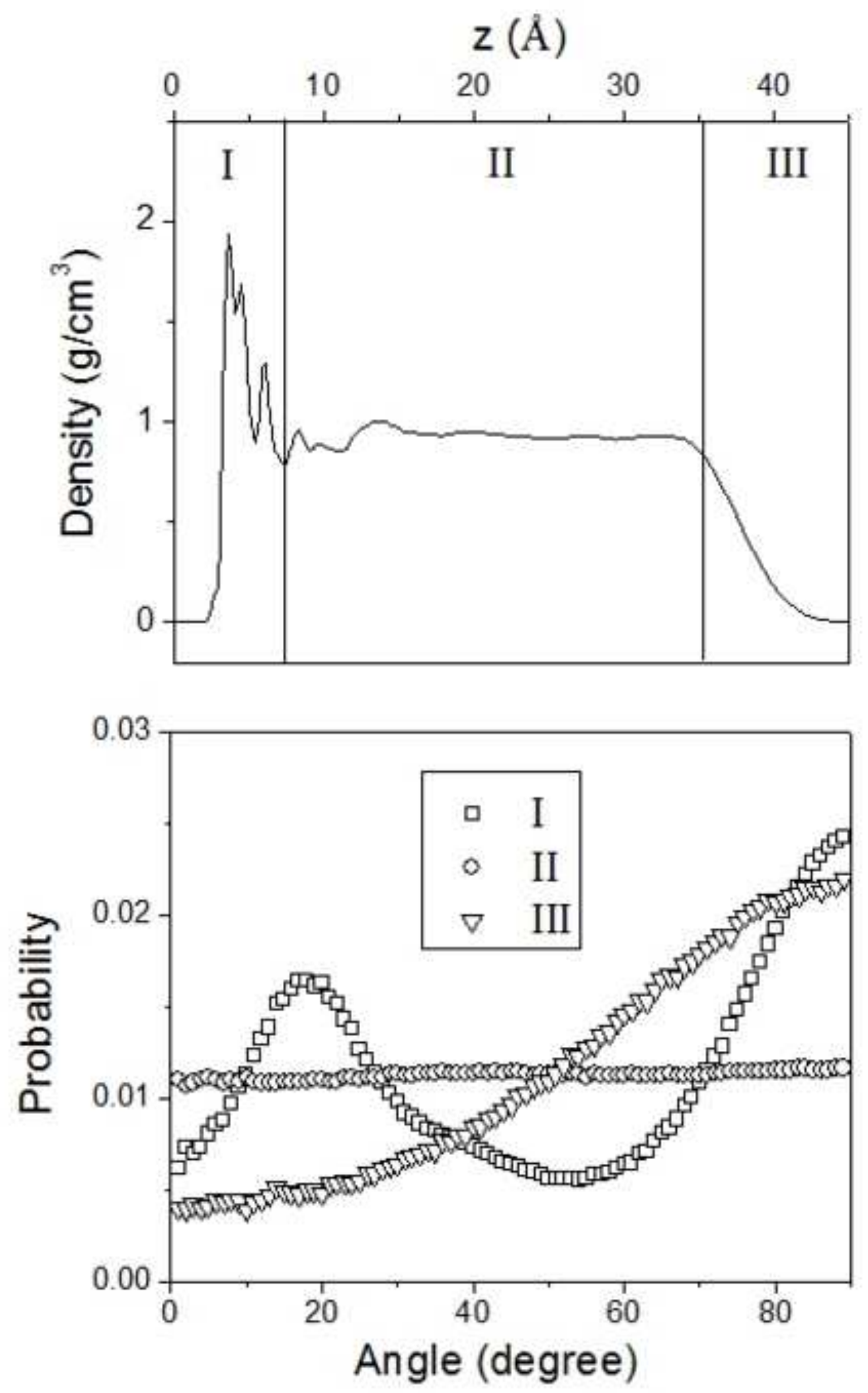


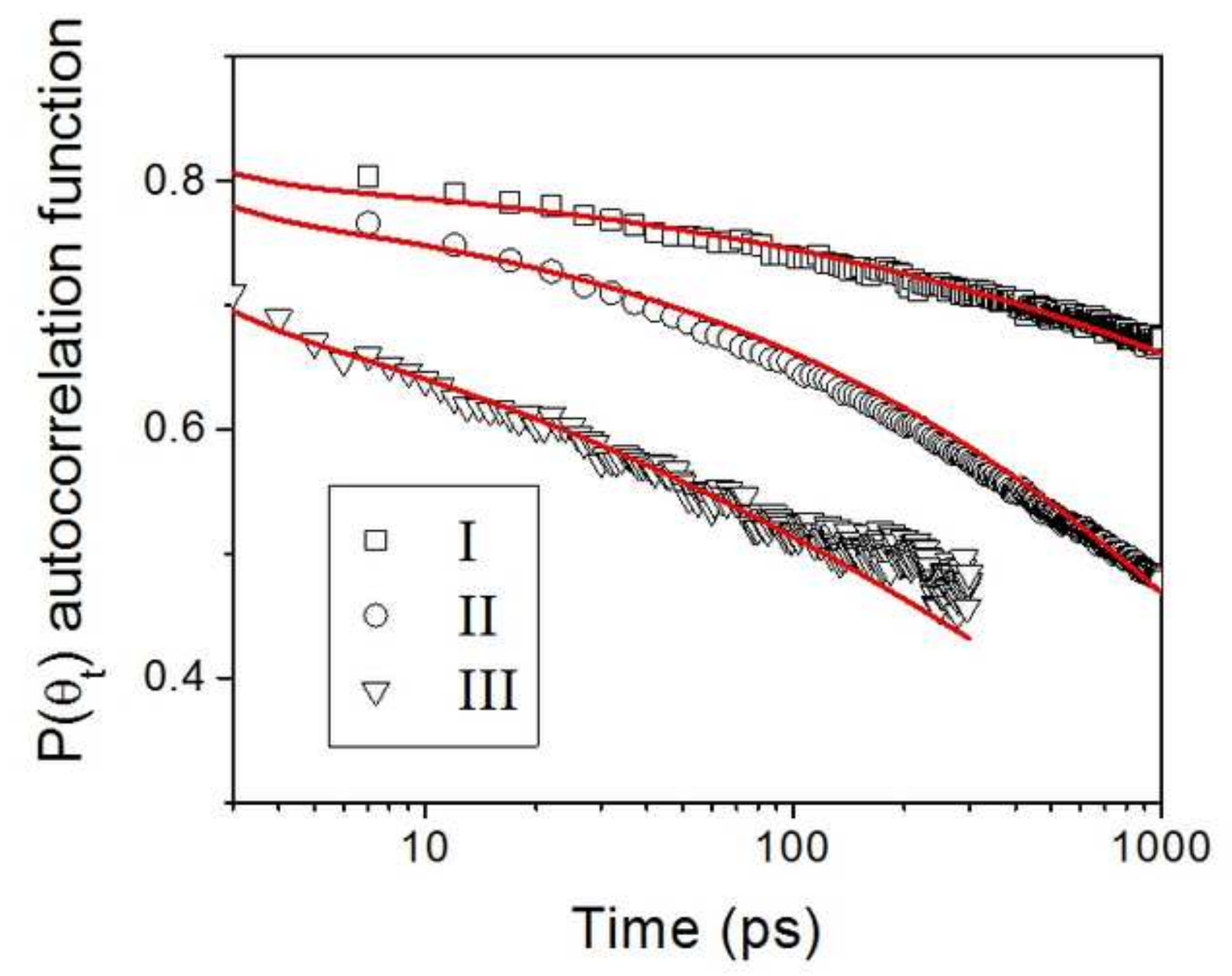

